\title{
ON DUBROVIN VALUATION RINGS IN CROSSED PRODUCT ALGEBRAS
}

\author{
DARRELL HAILE AND PATRICK MORANDI
}

\begin{abstract}
Let $F$ be a field and let $V$ be a valuation ring in $F$. If $A$ is a central simple $F$-algebra then $V$ can be extended to a Dubrovin valuation ring in $A$. In this paper we consider the structure of Dubrovin valuation rings with center $V$ in crossed product algebras $(K / F, G, f)$ where $K / F$ is a finite Galois extension with Galois group $G$ unramified over $V$ and $f$ is a normalized two-cocycle. In the case where $V$ is indecomposed in $K$ we introduce a family of orders naturally associated to $f$, examine their basic properties, and determine which of these orders is Dubrovin. In the case where $V$ is decomposed we determine the structure in the case of certain special discrete, finite rank valuations.
\end{abstract}

\section{0 . INTRODUCTION}

Let $V$ be a discrete valuation ring in a field $F$ and let $K / F$ be a finite unramified Galois extension with Galois group $G$ (say). Let $W$ be the integral closure of $V$ in $K$. In [H] the first author initiated a study of a certain family of orders over $V$ in crossed product algebras $(K / F, G, f)$. If the two-cocycle $f$ takes its values in $W$ then one can form in the obvious way a "crossed product order" $\sum W x_{\sigma} \subseteq \sum K x_{\sigma}=(K / F, G, f)$. It turns out that these orders have many interesting properties. For example they are primary with an explicitly described radical. Moreover they are a sufficiently large family to include, up to a suitable notion of equivalence, all of the maximal orders over $V$, in the case where the residue field of $V$ is perfect.

In 1982 Dubrovin in $\left[D_{1}\right]$ defined a notion of valuation ring inside an arbitrary simple Artinian ring. These Dubrovin valuation rings have many properties in common with maximal orders over discrete valuation rings. In particular the Dubrovin valuation rings with center a discrete valuation ring $V$ are precisely the maximal orders over $V$. In [MW] Morandi and Wadsworth investigated Dubrovin valuation rings over $V$ in $(K / F, G, f)$ where $V$ is an arbitrary valuation ring unramified and indecomposed in $K$. Among other things they showed that any Dubrovin valuation ring $B$ is integral over $V$ and that its residue ring $B / J(B)$ is a crossed product algebra. This work gave simpler proofs of many results on the structure of division algebras over henselian

Received by the editors September 4, 1990 and, in revised form, May 6, 1991.

1991 Mathematics Subject Classification. Primary 16H05; Secondary 13A20, 16 S35.

Supported in part by the National Science Foundation. Most of the research for this paper was done while the second author was a visitor at Indiana University. He wishes to thank the Mathematics Department there for its hospitality. 
valuation rings. If $V$ is discrete of rank one this case is the same as that considered in $\S 2$ of $[\mathrm{H}]$, but the viewpoint of the two papers is quite different.

In this paper we consider Dubrovin valuation rings over $V$ in a crossed product algebra $(K / F, G, f)$ where $V$ is any valuation ring of $F$ which is unramified in $K$. In doing so we combine the viewpoints of [H and $\mathrm{MW}$ ], and extend the results of both papers. Although even in the case of a perfect residue field the resulting orders do not include all of the Brauer classes as was the case in $[\mathrm{H}]$, the class of algebras we consider contains many interesting examples. In fact many constructions in the theory of simple algebras have used such examples.

To describe our results let $V$ be a valuation ring of a field $F$ and let $K / F$ be a Galois extension in which $V$ is unramified. Let $W$ be the integral closure of $V$ in $K$ and let $G=\operatorname{Gal}(K / F)$. Let $f \in Z^{2}\left(G, K^{\times}\right)$be a normalized twococycle. The first section of the paper contains some necessary preliminaries. In $\S 2$ we consider the case where $V$ is indecomposed in $K$. As in $[\mathrm{H}]$ we introduce a special family of orders. Let $Y$ be a finite set of overrings of $V$, each overring properly contained in $F$, and assume $V \in Y$. We may write $Y=\left\{V_{1}, V_{2}, \ldots, V_{n}\right\}$ where $V=V_{n} \subseteq V_{n-1} \subseteq \cdots \subseteq V_{1} \subseteq V_{0}=F$. Each $V_{i}$ is indecomposed and unramified in $K$. Let $W_{i}$ be the unique extension of $V_{i}$ to $K$. Let $H_{i}=\left\{\sigma \in G \mid f\left(\sigma, \sigma^{-1}\right) \in W_{i}^{\times}\right\}$. We say $f$ is standard for $Y$ if for each $i, f\left(H_{i} \times H_{i}\right) \subseteq W_{i+1}$. It turns out that every cocycle is cohomologous to one that is standard for $Y$. Now assume $f$ is standard for $Y$. It turns out that in this case the sets $H_{i}$ are in fact subgroups of $G$. For each $\sigma \in G$ we select an overring $W_{\sigma}$ in $Y$ as follows: If $\sigma \in H_{n}$ we let $W_{\sigma}=W_{n}=W$. If $\sigma \in H_{i}-H_{i+1}$ where $i<n$ then we set $W_{\sigma}=W_{i+1}$. We then set $B_{f}=\sum W_{\sigma} x_{\sigma}$. It turns out that for every choice of $Y$ and every cocycle $f$ standard for $Y$ the set $B_{f}$ is a ring and in fact a $V$-order in $(K / F, G, f)$. We call this the standard crossed product order for $f$ (and $Y$ ). This family of orders is very well behaved. For example each $B_{f}$ is a primary ring with an easily described radical. Part of the interest in this family lies in the fact that in the case where $V$ is discrete and finite rank any Dubrovin valuation ring over $V$ in $(K / F, G, f)$ is conjugate to such an order. The main results in $\S 2$ are Theorems 2.14 and 2.17 in which we determine which standard crossed product orders are Dubrovin valuation rings. The determination is in terms of conditions on the values of the cocycle and the relations between the subgroups $H_{i}$. To give an idea of the sort of conditions that arise consider the special case where $Y$ consists of $V$ alone. If $f$ is standard for $Y$ then there is only one nontrivial subgroup $H=\left\{\sigma \in G \mid f\left(\sigma, \sigma^{-1}\right) \in W^{\times}\right\}$. As in $[\mathrm{H}]$ we introduce the partial order on the coset space $G / H$ given by $\sigma H \leq \tau H$ if $f\left(\sigma, \sigma^{-1} \tau\right) \in W^{\times}$. In Theorem 2.14 we prove that the corresponding order $B_{f}=\sum W x_{\sigma}$ is a Dubrovin valuation ring over $V$ if and only if $H$ is a normal subgroup with cyclic quotient and there is a distinguished generator $\sigma H$ of $G / H$ satisfying two conditions: $f\left(\sigma, \sigma^{-1}\right) \in J(W)-J(W)^{2}$ and the partial order is the chain $H \leq \sigma H \leq \sigma^{2} H \leq \cdots \leq \sigma^{k-1} H$ where $k=|G / H|$. This is a generalization of Theorem 2.3 of $[\mathrm{H}]$. The arguments make heavy use of the notion of a value function as introduced by Morandi in [M].

In the third section we consider the case where $V$ is unramified in $K$ but not necessarily indecomposed. This case turns out to be considerably more 
complicated and we restrict our attention to those valuations that are discrete of finite rank. Let $W$ be the integral closure of $V$ in $K$. It turns out once again that a Dubrovin valuation ring in the crossed product algebra $(K / F, G, f)=$ $\sum K x_{\sigma}$ is conjugate to an algebra of the form $B=\sum W_{\sigma} x_{\sigma}$ where each $W_{\sigma}$ is an overring of $W$ contained in $K$. The aim is to determine the $W_{\sigma}$ and to see what one can say about the group $G$ and the values of $f$. Part of the difficulty arises from the fact that unlike the indecomposed case $B$ is not necessarily integral and so the theory of value functions does not apply. Each $W_{\sigma}$ is a Prüfer ring and thus equal to the intersection of the valuation rings that contain it. We first prove that the determination of the rings $W_{\sigma}$ can be reduced to the determination of the single ring $W_{\text {id }}$. We say a prime ideal $P$ of $W$ belongs to $B$ if $W_{\text {id }}$ is contained in the valuation ring $W_{P}$. If $P$ is any prime ideal of $W$ we let $D(P)$ denote its decomposition group and $H(P)=\left\{\sigma \in D(P) \mid f\left(\sigma, \sigma^{-1}\right) \notin P\right\}$. We prove two basic facts about these groups: If $P$ is a prime of height $i$ belonging to $B$ then $H(P)$ acts transitively on the set of primes of height $i+1$ that belong to $B$ and if $Q \supseteq P$ is a height $i+1$ prime belonging to $B$ then the group $H(Q)$ is normal in $D(Q) \cap H(P)$ with cyclic quotient generated by a coset $\sigma H(Q)$ satisfying two conditions similar to those described above. Again these results generalize $\S 3$ of $[\mathrm{H}]$. Along the way we once again give an explicit description of the radical of $B$. One of the ideas in the proofs is to show that one can find for each prime $P$ a Dubrovin valuation ring "related" to $B$ that satisfies the conditions of $\S 2$. This allows us to apply the results obtained there to $B$. We end with an example in rank 2 .

\section{Preliminaries}

We begin this section with a brief introduction to Dubrovin valuation rings. First recall that a ring $B$ is primary if the Jacobson radical $J(B)$ is a maximal ideal of $B$, that is $B / J(B)$ is simple. The ring $B$ is said to be Bezout if every finitely generated one-sided ideal of $B$ is principal. A Dubrovin valuation ring is a prime PI ring $B$ which is primary and Bezout. For brevity we will often refer to such a ring simply as a valuation ring. Let $S$ be the simple Artinian ring of quotients of $B$. It is shown in $\left[\mathrm{D}_{1}, \mathrm{D}_{2}\right]$ that $B Z(S)=S, B \cap Z(S)=V$ is a valuation ring of $Z(S)$, and two-sided ideals of $B$ are linearly ordered by inclusion, as are overrings of $B$ in $S$. Furthermore if $A$ is an overring of $B$ in $S$, then $A$ is a valuation ring, $A=B Z(A), J(A) \subseteq B$, and $B / J(A)$ is a valuation ring of $A / J(A)$. If $S$ is a central simple $F$-algebra and $V$ a valuation ring of $F$, it is shown in $\left[\mathrm{D}_{2}, \S 3\right.$, Theorem 2] and [BG, Theorem 3.8] that there is a valuation ring $B$ of $S$ with $B \cap F=V$. Also any two valuation rings of $S$ with center $V$ are conjugate [W, Theorem A]. For a fuller introduction to valuation rings see [W].

Let $V$ be a valuation ring of a field $F$ and $K$ a finite Galois extension of $F$ with Galois group $G$. Let $W$ be the integral closure of $V$ in $K$. In this paper we will only consider the case where $V$ is unramified in $K$, that is for all maximal ideals $M$ of $W$, the ramification index of $W_{M}$ over $V$ is one, the residue extension $W_{M} / J\left(W_{M}\right)$ over $V / J(V)$ is separable and $K / F$ is defectless with respect to $V$ (so $\sum_{M}\left[W_{M} / J\left(W_{M}\right): V / J(V)\right]=[K: F]$ ). It follows that $W_{M} / J\left(W_{M}\right)$ is Galois over $V / J(V)$ [E, 19.12]. If in addition $V$ is indecomposed in $K$, that is $W$ is a valuation ring, we say $K / F$ is inertial 
with respect to $V$. The ring $W$ will be of considerable importance in this paper, so we mention some properties that will be used throughout. Because $V$ is a valuation ring, $W$ is a Prüfer ring. Hence any localization of $W$ at a prime ideal is a valuation ring, and the extensions of $V$ to $K$ are precisely the localizations of $W$ at its maximal ideals. Furthermore because $K / F$ is finite, $W$ is semilocal. Every overring of $W$ in $K$ is also Prüfer and is a finite intersection of localizations of $W$. Proofs of these statements can be found in $[\mathrm{E}, 11.9,13.4,13.7]$.

Let $f \in Z^{2}\left(G, K^{\times}\right)$be a normalized two-cocycle and $\Sigma=(K / F, G, f)$ the corresponding crossed product algebra. Thus we have $\Sigma=\sum_{\sigma \in G} K x_{\sigma}$ where multiplication is given by $x_{\sigma} a=\sigma(a) x_{\sigma}$ for all $a \in K$ and $x_{\sigma} x_{\tau}=f(\sigma, \tau) x_{\sigma \tau}$ for all $\sigma, \tau \in G$. With $K$ as above, if $B$ is a valuation ring of $\Sigma$ lying over $V$ then we want to show that a suitable conjugate of $B$ can be written in a form compatible with the decomposition $\Sigma=\sum_{\sigma \in G} K x_{\sigma}$ and that the precise structure of $B$ can be obtained from $K / F$ and $f$. The following lemmas give the foundations for determining $B$.

Lemma 1.1. If $V$ is unramified in $K$ then for every $\sigma \in G-\{1\}$ and every maximal ideal $M$ of $W$ there is an $x \in W$ such that $\sigma(x)-x \notin M$.

Proof. Let $D(M)=\{\sigma \in G \mid \sigma(M)=M\}$, the decomposition group of $M$. If $\sigma \notin D(M)$ then $\sigma(M) \neq M$, so there is an $x \in M$ such that $\sigma(x) \notin M$ and so $\sigma(x)-x \notin M$. Now suppose $\sigma \in D(M)$. Because $K / F$ is unramified, the inertia group $I(M)=\left\{\sigma \in D(M) \mid \overline{\sigma(x)}=\bar{x}\right.$ for all $\left.x \in W_{M}\right\}$ is trivial, where $W_{M}$ is the localization of $W$ at $M$ and $\bar{x}=x+J\left(W_{M}\right)$. Thus if $\sigma \neq 1$, there is an $x \in W$ such that $\overline{\sigma(x)} \neq \bar{x}$, hence $\sigma(x)-x \notin M$.

It follows from this lemma and [DI, Chapter III, Theorem 1.1] that $W / V$ is a Galois extension of rings.

Lemma 1.2. The ring $W$ is a finitely generated $V$-module. There is a valuation ring $B$ of $\Sigma$ lying over $V$ with $W \subseteq B$.

Proof. Because $K / F$ is unramified, hence defectless, we have

$$
\sum_{M}\left[W_{M} / J\left(W_{M}\right): V / J(V)\right]=[K: F]
$$

Thus by [E, 18.6] $W$ is a finite $V$-module.

Let $B$ be a valuation ring of $\Sigma$ lying over $V$. Because $B F=\Sigma$ and $B$ is a Bezout ring, the finitely generated $B$-module $W B$ is principal, say $W B=x B$. Because $1 \in W B$, we have $x \in \Sigma^{\times}$. Thus $x B=W B=W(W B) \supseteq W x$, so $W \subseteq x B x^{-1}$, another valuation ring lying over $V$.

This lemma was discovered independently by Westmoreland [We].

If $B$ is a valuation ring of $\Sigma$ containing $W$ then $B$ is a $W-W$ submodule of $\Sigma$. The following lemma is the first step towards describing $B$.

Lemma 1.3. If $T$ is a $W-W$ submodule of $\Sigma$ then $T=\sum_{\sigma \in G}\left(T \cap K x_{\sigma}\right)$. In particular if $B$ is a valuation ring of $\Sigma$ lying over $V$ and containing $W$, then $B=\sum_{\sigma \in G} I_{\sigma} x_{\sigma}$, where each $I_{\sigma}$ is a $W$-submodule of $K$.

Proof. Let $T_{\sigma}=\left\{a \in K \mid a x_{\sigma} \in T\right\}$, a $W$-submodule of $K$. Clearly $\sum T_{\sigma} x_{\sigma} \subseteq$ $T$. To show equality let $\sum_{\sigma} a_{\sigma} x_{\sigma} \in T$. We need to show $a_{\sigma} x_{\sigma} \in T$ for all $\sigma$. Suppose this is false and that $r$ is minimal with $t=\sum_{i=1}^{r} a_{\sigma_{i}} x_{\sigma_{i}} \in T$, but 
not all $a_{\sigma_{i}}$ in $T_{\sigma_{i}}$. From the minimality it follows that $a_{\sigma_{i}} \notin T_{\sigma_{i}}$ for all $i$. Let $I=\left\{w \in W \mid w a_{\sigma_{1}} x_{\sigma_{1}} \in T\right\}$, an ideal of $W$. Because $I \neq W$ there is a maximal ideal $M$ of $W$ such that $I \subseteq M$. By Lemma 1.1 there is a $u \in W$ such that $\sigma_{1}(u)-\sigma_{2}(u) \notin M$. Thus

$$
\begin{aligned}
\sigma_{2}(u) t-t u= & \left(\sigma_{2}(u)-\sigma_{1}(u)\right) a_{\sigma_{1}} x_{\sigma_{1}}+\left(\sigma_{2}(u)-\sigma_{3}(u)\right) a_{\sigma_{3}} x_{\sigma_{3}} \\
& +\cdots+\left(\sigma_{2}(u)-\sigma_{r}(u)\right) a_{\sigma_{r}} x_{\sigma_{r}} \in T .
\end{aligned}
$$

By the minimality of $r$ we obtain $\left(\sigma_{2}(u)-\sigma_{1}(u)\right) a_{\sigma_{1}} x_{\sigma_{1}} \in T$, so $\left(\sigma_{2}(u)-\sigma_{1}(u)\right) \in$ $I \subseteq M$, a contradiction.

From these lemmas we see that there is a valuation ring $B$ of $\Sigma$ that contains $W$ and so decomposes into $B=\sum_{\sigma \in G} I_{\sigma} x_{\sigma}$. The task of describing $B$ thus reduces to describing the $I_{\sigma}$. The following simple lemma will be used repeatedly.

Lemma 1.4. If $\sigma \in G$, then $I_{\sigma} I_{\sigma^{-1}}^{\sigma} f\left(\sigma, \sigma^{-1}\right) \subseteq I_{\mathrm{id}}$. If all the $I_{\sigma}$ are rings, then $f\left(\sigma, \sigma^{-1}\right) \in I_{\text {id }}$.

Proof. Because $B$ is a ring, $\left(I_{\sigma} x_{\sigma}\right)\left(I_{\sigma^{-1}} x_{\sigma^{-1}}\right) \subseteq I_{\text {id }}$. Because $x_{\sigma} x_{\sigma^{-1}}=f\left(\sigma, \sigma^{-1}\right)$ we see that $I_{\sigma} I_{\sigma-1}^{\sigma} f\left(\sigma, \sigma^{-1}\right) \subseteq I_{\text {id }}$. If all the $I_{\sigma}$ are rings, then $f\left(\sigma, \sigma^{-1}\right) \in$ $I_{\sigma} I_{\sigma^{-1}}^{\sigma} f\left(\sigma, \sigma^{-1}\right) \subseteq I_{\text {id }}$.

Notice that this lemma implies that $I_{\mathrm{id}}$ is an overring of $W$ and that for all $\sigma \in G$ and all $b \in I_{\sigma^{-1}}^{\sigma}$ we have $I_{\sigma} b f\left(\sigma, \sigma^{-1}\right) \subseteq I_{\text {id }}$, so in particular $I_{\sigma}$ is a fractional ideal over $I_{\text {id }}$. In the case where $V$ is a discrete valuation ring the valuation ring $B$ is necessarily finitely generated as a $V$-module. It follows that $I_{\text {id }}=W$ and each $I_{\sigma}$ is a finitely generated $W$-submodule of $K$ and so principal over $W$, because $W$ is a principal ideal domain. If $I_{\sigma}=c_{\sigma} x_{\sigma}$ for all $\sigma \in G$, then by replacing $f$ by the equivalent cocylce $g$ corresponding to replacing $x_{\sigma}$ by $c_{\sigma} x_{\sigma}$ we can assume $B=\sum_{\sigma \in G} W x_{\sigma}$. For general valuation rings such a nice decomposition is not always possible (see Example 2.18). However in a number of situations one can considerably simplify the decomposition of $B$.

Suppose $V$ is a valuation ring of $F$ whose associated value group is $\mathbb{Z} \oplus \cdots \oplus \mathbb{Z}$ ( $n$ times) ordered antilexiographically. Then the Krull dimension of $V$ is $n$, and the overrings of $V$ in $F$ are $V=V_{n} \subseteq V_{n-1} \subseteq \cdots \subseteq V_{1} \subseteq F$ where $V_{i}$ is a valuation ring with value group $\mathbb{Z}^{i}$ (so $\bar{V}_{1}$ is a discrete valuation ring) and $V_{i+1} / J\left(V_{i}\right)$ is a discrete valuation ring of the field $V_{i} / J\left(V_{i}\right)$. We will call $V$ a discrete rank $n$ valuation ring. For such a $V$ not all $V$-submodules of $F$ are principal over $V$, but any such module is necessarily principal over $V_{i}$ for some $i$, as the following lemma shows.

Lemma 1.5. Let $V$ be a discrete rank $n$ valuation ring of $F$. If $I$ is a $V$ submodule of $F$, then $I=c U$ for some overring $U$ of $V$.

Proof. We use induction on $n=\operatorname{dim}(V)$. If $n=1$ then $V$ is a discrete valuation ring and the result is well known. So suppose $n>1$. If $I=F$ we are done, so assume $I \neq F$. If $V_{1} \supseteq V$ is the rank one overring of $V$, then $V_{1}$ is a discrete valuation ring, so $I V_{1}=d V_{1}$ for some $d \in F^{\times}$. By replacing $I$ by $d^{-1} I$ we may assume $I V_{1}=V_{1}$. Then by the linear order of $V$-submodules we see that $J\left(V_{1}\right) \subseteq I$. Thus $I / J\left(V_{1}\right)$ is a $V / J\left(V_{1}\right)$-submodule of $V_{1} / J\left(V_{1}\right)$. By the induction hypothesis $I / J\left(V_{1}\right)=\bar{c} T$ for some overring $T$ of $V / J\left(V_{1}\right)$. 
Let $U=\left\{x \in V_{1} \mid \bar{x} \in T\right\}$, an overring of $V$. If $c \in V_{1}$ is any preimage of $\bar{c}$, then $c$ is a unit in $V_{1}$ and so $J\left(V_{1}\right) \subseteq c U$. Hence $I=c U$.

Lemma 1.6. Let $V$ be a discrete rank $n$ valuation ring of $F, K / F$ a finite extension and $W$ the integral closure of $V$ in $K$. If $S$ is an overring of $W$ in $K$ and $I$ is an $S$-submodule of $K$, then $I=c U$ for some overring $U$ of $S$.

Proof. By [E, 13.7] $S$ has only finitely many maximal ideals, say $M_{1}, \ldots, M_{r}$. Because $I$ is a torsion-free $S$-module, $I=\bigcap_{i} I S_{M_{i}}$. By Lemma 1.5, $I S_{M_{i}}=$ $c_{i} U_{i}$ for some overring $U_{i}$ of $S_{M_{i}}$. By relabeling if necessary we may assume $U_{1}, \ldots, U_{t}$ are the minimal rings among the $U_{i}$. Then $I=\bigcap_{i=1}^{t} I U_{i}$. We want to apply Ribenboim's approximation theorem [R, $\S \mathrm{E}$, Theorem 3] to obtain an element $x \in K$ such that $x \equiv c_{i} \bmod c_{i} J\left(U_{i}\right), 1 \leq i \leq t$. To do so we need to show $c_{i}-c_{j} \in c_{i} J\left(U_{i}\right) U_{i j}=c_{j} J\left(U_{j}\right) U_{i j}$ for $i \neq j$, where $U_{i j}=U_{i} U_{j}$. Because the $U_{i}$ are pairwise incomparable, $U_{i}$ and $U_{j}$ are proper subrings of $U_{i j}$ and so

$$
c_{i} J\left(U_{i}\right) U_{i j}+c_{i} U_{i j}=I U_{i j}=c_{j} U_{i j}=c_{j} J\left(U_{j}\right) U_{i j}
$$

and $c_{i}-c_{j} \in I U_{i j}$. Hence such an $x$ exists and so for each $i, x=c_{i}\left(1+m_{i}\right)$ for some $m_{i} \in J\left(U_{i}\right)$. Thus $x U_{i}=c_{i} U_{i}$. Therefore we obtain

$$
I=\bigcap_{i=1}^{t} I U_{i}=\bigcap c_{i} U_{i}=\bigcap x U_{i}=x\left(\bigcap U_{i}\right)=x U,
$$

where $U$ is the overring $\cap U_{i}$.

We now summarize what we have learned about valuation rings over discrete rank $n$ valuation rings in crossed product algebras.

Proposition 1.7. Let $V$ be a discrete rank $n$ valuation ring in a field $F$. Let $K / F$ be a finite Galois extension in which $V$ is unramified and let $G=\mathrm{Gal}(K / F)$. Let $W$ be the integral closure of $V$ in $K$ and let $f \in Z^{2}\left(G, K^{\times}\right)$be a normalized two-cocycle. There is a valuation ring $B$ in $(K / F, G, f)$ and a cocycle $f^{\prime}$ cohomologous to $f$ such that if $\left(K / F, G, f^{\prime}\right)=\sum K x_{\sigma}$ then $B=\sum W_{\sigma} x_{\sigma}$ where $W_{\mathrm{id}}$ is an overring of $W$ and each $W_{\sigma}$ is an overring of $W_{\mathrm{id}}$.

Proof. We have already seen that there is a valuation ring $B$ in $(K / F, G, f)=$ $\sum K y_{\sigma}$ such that $B=\sum I_{\sigma} y_{\sigma}$ where $I_{\text {id }}$ is an overring of $W$ and each $I_{\sigma}$ is an $I_{\text {id }}$-submodule of $K$. By Lemma 1.6 we can write $I_{\sigma}=c_{\sigma} W_{\sigma}$ where $c_{\sigma} \in K$ and $W_{\sigma}$ is an overring of $W_{\text {id }}=I_{\text {id }}$. Hence by replacing $f$ by the cocycle $f^{\prime}$ corresponding to replacing $y_{\sigma}$ by $x_{\sigma}=c_{\sigma} y_{\sigma}$ we obtain the desired form.

\section{INDECOMPOSED CASE}

In this section we consider the case where the valuation ring $V$ is indecomposed and unramified in $K$. For each cocylce we will construct a natural set of orders which in the discrete, finite rank case contains a valuation ring for that cocycle. We will also show how to determine which of these orders is a valuation ring.

Let $Y$ be a finite set of overrings of $V$, each properly contained in $F$, and assume $Y$ contains $V$. Because the overrings are linearly ordered we may write $Y=\left\{V_{1}, V_{2}, \ldots, V_{n-1}, V_{n}\right\}$ where $V=V_{n} \subseteq V_{n-1} \subseteq \cdots \subseteq V_{1} \subseteq V_{0}=F$. Because $V$ is indecomposed and unramified in $K$, it follows that each $V_{i}$ is 
also indecomposed and unramified in $K$. Let $W_{i}$ be the unique extension of $V_{i}$ to $K$ (and let $W_{0}=K$ ). Let $f: G \times G \rightarrow K^{\times}$be a (normalized) two-cocycle. For each $i, 0 \leq i \leq n$, let $H_{i}=\left\{\sigma \in G \mid f\left(\sigma, \sigma^{-1}\right) \in W_{i}^{\times}\right\}$(note that $H_{0}=G$ ).

Definition. The cocycle $f$ is said to be standard for $Y$ if for all $i, 0 \leq i \leq n-1$, $f\left(H_{i} \times H_{i}\right) \subseteq W_{i+1} \quad$ and so $\left.\subseteq W_{i+1} \backslash J\left(W_{i}\right)\right)$.

Lemma 2.1. If $f$ is a standard cocycle for $Y$, then for all $i, 0 \leq i \leq n, H_{i}$ is a subgroup of $G$.

Proof. We proceed by induction on $i$. If $i=0$ there is nothing to prove. Now assume $i>0$. Let $\sigma, \tau \in H_{i}$. We have the cocycle identities:

$$
\begin{aligned}
& f^{\sigma}\left(\sigma^{-1}, \tau\right) f\left(\sigma, \sigma^{-1} \tau\right)=f\left(\sigma, \sigma^{-1}\right), \\
& f^{\tau}\left(\tau^{-1}, \sigma\right) f\left(\tau, \tau^{-1} \sigma\right)=f\left(\tau, \tau^{-1}\right), \\
& f^{\sigma}\left(\sigma^{-1} \tau, \tau^{-1} \sigma\right)=f\left(\sigma, \sigma^{-1} \tau\right) f\left(\tau, \tau^{-1} \sigma\right) .
\end{aligned}
$$

Because $H_{i} \subseteq H_{i-1}$ and $H_{i-1}$ is a subgroup by induction, all the values in these identities lie in $W_{i}$. Because $f\left(\sigma, \sigma^{-1}\right), f\left(\tau, \tau^{-1}\right)$ are units in $W_{i}$, it follows that $f^{\sigma}\left(\sigma^{-1} \tau, \tau^{-1} \sigma\right)$ is a unit, so $\sigma^{-1} \tau \in H_{i}$. Hence $H_{i}$ is a subgroup of $G$.

We want to show that every cocycle is equivalent to a standard one. We need the following lemma.

Lemma 2.2. Let $K / F$ be a finite Galois extension with Galois group $G$. Let $R_{2} \subseteq R_{1}$ be valuation rings with field of fractions $F$ and assume $R_{2}$ is unramified and indecomposed in $K$. Let $S_{i}$ be the extension of $R_{i}$ in $K, i=1,2$. Let $f: G \times G \rightarrow S_{1}^{\times}$be a cocycle. Then there is a cocycle $g$ equivalent to $f$ over $S_{1}^{\times}$such that $g(G \times G) \subseteq S_{2}$.

Proof. Because $S_{2}$ is a valuation ring the fractional ideal $I$ generated by the $f(\sigma, \tau), \sigma, \tau \in G$, is principal, that is $I=a S_{2}$ for some $a \in K$. Because $f(G \times G) \subseteq S_{1}^{\times}$, we have $I S_{1}=S_{1}$ and so $a \in S_{1}^{\times}$. Define a one-cochain $\alpha$ by $\alpha(1)=1$ and $\alpha(\sigma)=a^{-1}$ for $\sigma \in G-\{1\}$. An easy calculation shows that $g=(\partial \alpha) f$ has the desired property.

Proposition 2.3. Every two-cocycle is equivalent to one that is standard for $Y$.

Proof. Let $f=f_{0}$. We will construct a sequence of cocycles $f_{1}, f_{2}, \ldots, f_{n}$ such that for each $i, 1 \leq i \leq n-1$, the following two properties are satisfied.

(1) The cocycle $f_{i}$ is equivalent to $f_{i-1}$ over $W_{i-1}^{\times}$, that is there is a onecochain $\alpha: G \rightarrow W_{i-1}^{\times}$such that $f_{i}=(\partial \alpha) f_{i-1}$.

(2) We have $f_{i}\left(H_{i-1} \times H_{i-1}\right) \subseteq W_{i}$, where $H_{i-1}=\left\{\sigma \in G \mid f_{i}\left(\sigma, \sigma^{-1}\right) \in\right.$ $\left.W_{i-1}^{\times}\right\}$.

It will then follow that $f_{n}$ is equivalent to $f$ and standard for $Y$.

To do the first step of the construction, apply Lemma 2.2 to obtain a cocycle $f_{1}$ equivalent to $f$ over $K^{\times}$with $f_{1}(G \times G) \subseteq W_{1}$. Then $f_{1}$ is standard for $W_{1}$. This finishes the first step of the construction.

Now let $i$ be chosen, $1 \leq i \leq n-1$, and assume we have constructed $f_{1}, f_{2}, \ldots, f_{i}$ satisfying the two properties. We show how to construct $f_{i+1}$. We have $f_{i}$ is standard for $W_{i}$ and so by Lemma $2.1, H_{1}, H_{2}, \ldots, H_{i}$ are subgroups of $G$. Let $L$ be the fixed field of $H_{i-1}$. We apply Lemma 2.2 to the 
Galois extension $K / L$ and the valuation rings $W_{i+1} \cap L \subseteq W_{i} \cap L$. We obtain a cocycle $f_{i+1}$ equivalent to $f_{i}$ over $W_{i}^{\times}$such that $f_{i+1}\left(H_{i} \times H_{i}\right) \subseteq W_{i+1}$.

Let $f$ be a standard cocycle for $Y$. Let $i$ be an integer, $i \leq n-1$. Let $H=H_{i}$. The field extension $K / K^{H}$ is Galois with group $H$. Moreover at the residue level the field $W_{1} / J\left(W_{1}\right)$ is a Galois extension of the field $W_{1}^{H} / J\left(W_{1}\right)^{H}$ with Galois group $H$ and $W_{n}^{H} / J\left(W_{1}\right)^{H}$ is a valuation ring in $W_{1}^{H} / J\left(W_{1}\right)^{H}$. The following proposition is clear.

Proposition 2.4. Let $f$ be standard for $Y$.

(a) If $Y_{i}=\left\{V_{1}, V_{2}, \ldots, V_{i-1}, V_{i}\right\}$, then $f$ is standard for $Y_{i}$.

(b) If $Y^{\prime} \supseteq Y$ is a finite set of overrings of $V$, each properly contained in $F$, then $f$ is standard for $Y^{\prime}$.

(c) For all $i, i \leq n-1$, letting $H=H_{i}, f_{\mid H \times H}$ is standard for $\left\{W_{1}^{H} / J\left(W_{1}\right)^{H}\right.$, $\left.\ldots, W_{n}^{H} / J\left(W_{1}\right)^{H}\right\}$.

Now let $f$ be a cocylce that is standard for $Y=\left\{V_{1}, V_{2}, \ldots, V_{n-1}, V_{n}\right\}$. Let $\Sigma_{f}=\sum_{\sigma \in G} K x_{\sigma}$ be the central simple crossed product algebra over $F$ corresponding to $f$. For each $\sigma \in G$, either $\sigma \in H_{n}$ or there is a unique integer $i, 1 \leq i \leq n$, such that $\sigma \in H_{i-1}-H_{i}$. We will call $i$ the height of $\sigma$ (so we do not define the height for elements of $H_{n}$ ). If $\sigma \in H_{n}$ we let $W_{\sigma}=W_{n}$ and if $\sigma \notin H_{n}$ we let $W_{\sigma}=W_{i}$, where $i$ is the height of $\sigma$. Form the subset $B_{f}$ of $\Sigma_{f}$ given by $B_{f}=\sum_{\sigma \in G} W_{\sigma} x_{\sigma}$. Note that $B_{f}$ depends on $Y$ and not just on $f$ but the notation should not be confusing.

Recall that a $V$-subalgebra $R$ of $\Sigma$ is called an order over $V$ if $R F=\Sigma$ and $R$ is integral over $V$.

Proposition 2.5. Let $f$ be a standard cocycle for $Y$. The set $B_{f}$ is an order over $V$ in $\Sigma_{f}$.

Proof. We have to show $B_{f}$ is a ring and integral over $V$ (it is then clear that it is an order).

To show that $B_{f}$ is a ring it suffices to show that if $\sigma, \tau \in g$ then $W_{\sigma} x_{\sigma} W_{\tau} x_{\tau}$ $\subseteq W_{\sigma \tau} x_{\sigma \tau}$. This in turn reduces to showing that $W_{\sigma} W_{\tau} f(\sigma, \tau) \subseteq W_{\sigma \tau}$. So let $\sigma, \tau \in G$. If $\sigma, \tau \in H_{n}$ the $\sigma \tau \in H_{n}$ and $W_{\sigma}=W_{\tau}=W_{\sigma \tau}=W_{n}$. Moreover $f(\sigma, \tau) \in W_{n}$, so the desired inclusion holds. If exactly one of $\sigma, \tau$ is in $H_{n}$, say $\sigma \in H_{n}$, then $\sigma \tau \notin H_{n}$ and the elements $\tau$ and $\sigma \tau$ have the same height. It follows that $W_{\sigma} \subseteq W_{\sigma \tau}$ and $W_{\tau}=W_{\sigma \tau}$ and $f(\sigma, \tau) \in W_{\sigma \tau}$, so again the inclusion holds.

So we now may assume neither $\sigma$ nor $\tau$ lies in $H_{n}$. Let $i$ be the height of $\sigma$, and let $j$ be the height of $\tau$. Hence $W_{\sigma}=W_{i}$ and $W_{\tau}=W_{j}$. The argument breaks up into cases.

First assume $i>j$. Then $\sigma \tau \in H_{j-1}-H_{j}$, so $W_{\sigma \tau}=W_{j}$. Moreover $f(\sigma, \tau) \in W_{j}$ because $\sigma, \tau \in H_{j-1}$. The inclusion $W_{\sigma} W_{\tau} f(\sigma, \tau) \subseteq W_{\sigma \tau}$ is then clear.

The case $j>i$ is handled in the same way.

Now assume $i=j$. If $\sigma \tau \notin H_{i}$ then $W_{\sigma}=W_{\tau}=W_{\sigma \tau}=W_{i}$ and $f(\sigma, \tau) \epsilon$ $W_{i}$, so again the inclusion is clear.

Finally assume $i=j$ and $\sigma \tau \in H_{i}$. In that case (i.e., height $(\sigma \tau)>i$ ) $W_{\sigma}=W_{\tau}=W_{i}$, but $W_{\sigma \tau} \subseteq W_{i+1}$. However we claim that $f(\sigma, \tau) \in J\left(W_{i}\right)$. If 
so then

$$
W_{\sigma} W_{\tau} f(\sigma, \tau)=W_{i} f(\sigma, \tau) \subseteq J\left(W_{i}\right) \subseteq W_{n} \subseteq W_{\sigma \tau}
$$

as desired.

To see the claim we consider the following cocycle identity:

$$
f^{\sigma}\left(\tau, \tau^{-1} \sigma^{-1}\right) f\left(\sigma, \sigma^{-1}\right)=f(\sigma, \tau) f\left(\sigma \tau, \tau^{-1} \sigma^{-1}\right) .
$$

We know $f^{\sigma}\left(\tau, \tau^{-1} \sigma^{-1}\right) \in W_{i}, f\left(\sigma, \sigma^{-1}\right) \in J\left(W_{i}\right)$, and $f\left(\sigma \tau, \tau^{-1} \sigma^{-1}\right) \in$ $W_{i}^{\times}$. It follows that $f(\sigma, \tau) \in J\left(W_{i}\right)$.

It remains to show that the elements of $B_{f}$ are integral over $V$. By [AS, Theorem 2.3] it suffices to show that $B_{f}$ is generated as a $V$ module by integral elements. Hence it is enough to show that for each $\sigma \in G$, the set $W_{\sigma} x_{\sigma}$ consists of integral elements. Let $a \in W_{\sigma}$. Let $k$ be the order of $\sigma$ in $G$. Consider

$$
\left(a x_{\sigma}\right)^{k}=a \sigma(a) \sigma^{2}(a) \cdots \sigma^{k-1}(a) f(\sigma, \sigma) f\left(\sigma^{2}, \sigma\right) \cdots f\left(\sigma^{k-1}, \sigma\right) .
$$

If $\sigma \in H_{n-1}$ then $W_{\sigma}=W_{n}$ and $f\left(\sigma^{m}, \sigma\right) \in W_{n}$ for all integers $m$, so $\left(a x_{\sigma}\right)^{k} \in W_{n}$. But $W_{n}$ is integral over $V$ (in fact, finitely generated) and so $a x_{\sigma}$ is integral over $V$.

So assume $\sigma \notin H_{n-1}$ and let $i$ be the height of $\sigma$ (so $\left.i<n\right)$. Then $W_{\sigma}=W_{i}$ and $f\left(\sigma^{m}, \sigma\right) \in W_{i}$ for all integers $m$. Moreover,

$$
f\left(\sigma^{k-1}, \sigma\right)=f\left(\sigma^{-1}, \sigma\right)=f^{\sigma^{-1}}\left(\sigma, \sigma^{-1}\right) \in J\left(W_{i}\right) .
$$

Hence $\left(a x_{\sigma}\right)^{k} \in J\left(W_{i}\right) \subseteq W_{n}$, so $a x_{\sigma}$ is integral over $V$.

We will refer to $B_{f}$ as the crossed product order for $f$ (corresponding to $Y)$. We now want to derive the basic properties of these orders.

Proposition 2.6. Let $f$ be a standard cocycle for $Y$.

(a) The order $B_{f}$ is a primary ring with Jacobson radical

$$
J\left(B_{f}\right)=\sum_{\sigma \in H_{n}} J\left(W_{\sigma}\right) x_{\sigma}+\sum_{\sigma \notin H_{n}} W_{\sigma} x_{\sigma} .
$$

(b) For each $i, 1 \leq i \leq n-1$, let $B_{i}=B_{f} V_{i}$ (so $\left.B_{n}=B_{f}\right)$. Then $B_{i}$ is the crossed product order over $V_{i}$ corresponding to the standard cocycle $f$ for $Y_{i}=\left\{V_{j} \mid j \leq i\right\}$ and we have the inclusions $B_{f}=B_{n} \subseteq B_{n-1} \subseteq \cdots \subseteq B_{1}$.

(c) For each $i, 1 \leq i \leq n-1, J\left(B_{i}\right) \subseteq B_{f}$.

Proof. (a) We first show that

$$
I=\sum_{\sigma \in H_{n}} J\left(W_{\sigma}\right) x_{\sigma}+\sum_{\sigma \notin H_{n}} W_{\sigma} x_{\sigma}
$$

is an ideal in $B_{f}$. Let $I_{\sigma}=J\left(W_{\sigma}\right)$ for $\sigma \in H_{n}$ and let $I_{\sigma}=W_{\sigma}$ for $\sigma \notin H_{n}$, so that $I=\sum_{\sigma \in G} I_{\sigma} x_{\sigma}$. It suffices to show that for all $\sigma \in G, W_{\sigma} x_{\sigma} I_{\tau} x_{\tau} \subseteq I_{\sigma \tau} x_{\sigma \tau}$ and $I_{\tau} x_{\tau} W_{\sigma} x_{\sigma} \subseteq I_{\tau \sigma} x_{\tau \sigma}$. This reduces in turn to showing that $W_{\sigma} I_{\tau} f(\sigma, \tau) \subseteq$ $I_{\sigma \tau}$ and $I_{\tau} W_{\sigma} f(\tau, \sigma) \subseteq I_{\tau \sigma}$, where we are using the fact that for each $\sigma \in G$, the sets $I_{\sigma}$ and $W_{\sigma}$ are $G$-stable.

We will show $W_{\sigma} I_{\tau} f(\sigma, \tau) \subseteq I_{\sigma \tau}$. The argument for the other inclusion is similar and will be omitted. If $\sigma \tau \notin H_{n}$ then $I_{\sigma \tau}=W_{\sigma \tau}$ and the result follows from the fact that $B_{f}$ is a ring. So we may assume $\sigma \tau \in H_{n}$. In that case 
if $\sigma, \tau \in H_{n}$ then $W_{\sigma}=W_{n}, I_{\tau}=I_{\sigma \tau}=J\left(W_{n}\right)$, and $f(\sigma, \tau) \in W_{n}$ so the inclusion is clear. Finally assume neither $\sigma$ nor $\tau$ lies in $H_{n}$ but $\sigma \tau \in H_{n}$. It follows that $\sigma$ and $\tau$ must have the same height $i$ (say) and as we saw above in the proof of Proposition 2.5 this implies $f(\sigma, \tau) \in J\left(W_{i}\right)$. Hence

$$
W_{\sigma} I_{\tau} f(\sigma, \tau)=W_{i} f(\sigma, \tau) \subseteq J\left(W_{i}\right) \subseteq J\left(W_{n}\right)=I_{\sigma \tau}
$$

We show next that $I$ is maximal. Let $H=H_{n}$. Consider

$$
B_{f} / I=\sum_{\sigma \in G}\left(W_{\sigma} / I_{\sigma}\right) x_{\sigma}=\sum_{\sigma \in H} \bar{W}_{n} x_{\sigma}
$$

where $\bar{W}_{n}$ is the residue field of the valuation ring $W_{n}$. As we have seen $\bar{W}_{n}$ is a Galois extension of $\bar{W}_{n}^{H}$ with Galois group $H$ and so it is clear that $B_{f} / I$ is the crossed product algebra $\left(\bar{W}_{\sigma} / \bar{W}_{\sigma}^{H}, H, f_{\mid H \times H}\right)$. In particular $B_{f} / I$ is simple, so $I$ is maximal.

Finally we need to show $I$ is the unique maximal ideal of $B_{f}$. Suppose $T$ is another ideal and suppose $T$ is not contained in $I$. Then $I+T=B_{f}$. Because $T$ is a $W_{n}-W_{n}$ bimodule, we can apply Lemma 1.3 to write $T=\sum_{\sigma \in G} T_{\sigma} x_{\sigma}$, where $T_{\sigma} x_{\sigma}=T \cap W_{\sigma} x_{\sigma}$. Hence it follows that $T_{\text {id }}+I_{\text {id }}=W_{n}$. This means $T_{\text {id }}+J\left(W_{n}\right)=W_{n}$. But then it follows that $T_{\text {id }}=W_{n}$ and so $T=B_{f}$.

For (b) and (c), part (b) is clear and part (c) follows from the explicit description of $J\left(B_{i}\right)$ given in (a).

We now want to investigate the connection between the valuation rings in $\Sigma_{f}$ lying over $V$ and the orders we have introduced. Let $f: G \times G \rightarrow K^{\times}$be a normalized cocycle. Let $v$ be a valuation on $F$ corresponding to $V$ and let $\Gamma$ be the value group of $v$. Let $\Delta=\Gamma \otimes_{\mathbb{Z}} \mathbb{Q}$ be the divisible hull of $\Gamma$. As in [MW, p. 625], we define the function $w: \Sigma_{f} \rightarrow \Delta$ by $w\left(\sum_{\sigma} a_{\sigma} x_{\sigma}\right)=$ $\min _{\sigma}\left\{v\left(a_{\sigma}\right)+w\left(x_{\sigma}\right)\right\}$, where $v$ is used to denote the unique extension to $K$ and for each $\sigma \in G$,

$$
w\left(x_{\sigma}\right)=\frac{1}{n} \sum_{i=1}^{n-1} v\left(f\left(\sigma^{i}, \sigma\right)\right), \quad n=|G| .
$$

It is shown in [MW, Theorem 2.1] that because $K / F$ is inertial with respect to $v$ the function $w$ is a value function on $\Sigma_{f}$ and the set $B_{w}=\left\{s \in \Sigma_{f} \mid w(s) \geq 0\right\}$ is a valuation ring with Jacobson radical $J\left(B_{w}\right)=\left\{s \in \Sigma_{f} \mid w(s)>0\right\}$. It follows that $B_{w}=\sum_{\sigma \in G} N_{\sigma} x_{\sigma}$ where $N_{\sigma}=\left\{k \in K \mid w\left(k x_{\sigma}\right) \geq 0\right\}$. In particular $N_{\text {id }}=W$. For more details on value functions see [M, $\left.\S 2\right]$.

Observe that in the case where $V$ is a discrete rank $n$ valuation ring, it follows from Lemma 1.5 that each $N_{\sigma}$ is principal over an overring $W_{\sigma}$ of $W$. Hence by possibly changing to an equivalent cocycle $g$ we may assume $B_{w}=\sum_{\sigma \in G} W_{\sigma} x_{\sigma}$ where each $W_{\sigma}$ is an overring of $W_{\mathrm{id}}=W$. This motivates the following proposition.

Proposition 2.7. Let $V$ be a valuation ring of the field $F$ and let $K / F$ be an inertial Galois extension with Galois group $G$. Let $W$ be the extension of $V$ to $K$. Let $f \in Z^{2}\left(G, K^{\times}\right)$be a normalized two cocycle and let $B$ be a valuation ring in the crossed product algebra $\Sigma_{f}=\sum_{\sigma \in G} K x_{\sigma}$. If $B$ can be expressed as $B=\sum_{\sigma \in G} W_{\sigma} x_{\sigma}$ where each $W_{\sigma}$ is an overring of $W$, then $f$ is standard for $Y=\left\{W_{\sigma} \cap F \mid \sigma \in G\right\}$ and $B=B_{f}$. 
Proof. First observe that because $B$ is integral over $V$ [MW, Theorem 2.1] and $W_{\text {id }}$ is assumed to be an overring of $W$, it follows that $W_{\text {id }}=W$. We may label the elements of $Y$ so that $Y=\left\{V_{1}, V_{2}, \ldots, V_{n-1}, V_{n}\right\}$ where $V=V_{n} \subseteq$ $V_{n-1} \subseteq \cdots \subseteq V_{1} \subseteq F$. Let $W=W_{n} \subseteq W_{n-1} \subseteq \cdots \subseteq W_{1} \subseteq K$ be the extensions of the $V_{i}$ to $K$. For each $i, 0 \leq i \leq n$, let $H_{i}=\left\{\sigma \in G \mid f\left(\sigma, \sigma^{-1}\right) \in W_{i}^{\times}\right\}$. We now proceed by induction on $n$. If $n=1$ then $W_{\sigma}=W$ for all $\sigma$. The fact that $B$ is a ring then implies that $f(G \times G) \subseteq W$ and $B$ is clearly in standard form. Hence we may assume $n>1$. Let $B_{n-1}=B V_{n-1}=\sum_{\sigma \in G} W_{\sigma} V_{n-1} x_{\sigma}$. Then $B_{n-1}$ is a valuation ring and for each $\sigma, W_{\sigma} V_{n-1}$ is an overring of $W_{n-1}$. By the induction hypothesis we infer that $f$ is standard for $Y^{\prime}=Y-\{V\}$ and $B_{n-1}$ has the standard form. We claim that for all $\sigma \in H_{n-1}, W_{\sigma}=W_{n}$. If so then the fact that $B$ is a ring will imply that $f\left(H_{n-1} \times H_{n-1}\right) \subseteq W_{n}$ and that will complete the proof.

So let $\sigma \in H_{n-1}$. Because $B_{n-1}$ is in standard form, we know $W_{\sigma} V_{n-1}=$ $W_{n-1}$. Hence $W_{\sigma}=W_{n}$ or $W_{n-1}$. But $W_{\sigma} x_{\sigma} x_{\sigma^{-1}} \subset W_{\text {id }}=W_{n}$, so $W_{n} \supseteq$ $W_{\sigma} f\left(\sigma, \sigma^{-1}\right)$. If $W_{\sigma}=W_{n-1}$ then $W_{n} \supseteq W_{n-1} f\left(\sigma, \sigma^{-1}\right)=W_{n-1}$ because $f\left(\sigma, \sigma^{-1}\right) \in W_{n-1}^{\times}$. This is a contradiction so $W_{\sigma}=W_{n}$ as desired.

Using the observations made before this proposition we obtain the following consequence.

Corollary 2.8. Let $V, F, K$ and $f$ be as in the proposition with $V$ a discrete rank $n$ valuation ring. Let $Y=\{U \mid V \subseteq U \subset F$ and $U$ is a ring $\}$. If $B$ is a valuation ring in $\Sigma_{f}$, then there is a cocycle $g$ equivalent to $f$ such that $g$ is in standard form for $Y$ and $B$ is conjugate to $B_{g}$.

Proof. By Proposition 2.7 and the remarks preceding it there is a cocycle $g$ equivalent to $f$ such that $g$ is in standard form for some set of overrings $Y^{\prime}$ and $B$ is conjugate to $B_{g}$. However it then follows from part (b) of Proposition 2.4 that we may take $Y^{\prime}=Y$.

Having seen that at least in the discrete rank $n$ case every valuation ring is equivalent to one in standard form it is natural to try to characterize the valuation rings among the standard orders. As before let $f$ be a standard cocycle for $Y=\left\{V_{1}, V_{2}, \ldots, V_{n-1}, V_{n}\right\}$ and let $B_{f}$ be the corresponding crossed product order. For each $i, 1 \leq i \leq n-1$, we can (as in $[\mathrm{H}])$ introduce a partial ordering on the set of cosets $H_{i-1} / H_{i}$ as follows. If $\sigma, \tau \in H_{i-1}$ we define $\sigma H_{i} \leq \tau H_{i}$ if $f\left(\sigma, \sigma^{-1} \tau\right) \in W_{i}^{\times}$.

Lemma 2.9. Let $i$ be an integer, $1 \leq i \leq n-1$.

(a) The relation described above is well defined and gives a partial ordering on the set $H_{i-1} / H_{i}$.

(b) If $\sigma, \tau, \gamma \in H_{i-1}$ and $\sigma H_{i} \leq \gamma H_{i}$, then $\sigma H_{i} \leq \tau H_{i} \leq \gamma H_{i}$ if and only if $\sigma^{-1} \tau H_{i} \leq \sigma^{-1} \gamma H_{i}$.

Proof. (a) Let $\sigma, \tau \in H_{i-1}$. To show the relation is well defined it suffices to show that if $f\left(\sigma, \sigma^{-1} \tau\right) \in W_{i}^{\times}$and $h, k \in H_{i}$ then $f\left(\sigma h, h^{-1} \sigma^{-1} \tau k\right) \in W_{i}^{\times}$. We first observe that if $h \in H_{i}$ and $g \in H_{i-1}$, then $f(h, g), f(g, h) \in W_{i}^{\times}$: this follows from the identities

$$
f^{h^{-1}}(h, g) f\left(h^{-1}, h g\right)=f\left(h^{-1}, h\right) \text { and } f^{g}\left(h, h^{-1}\right)=f(g, h) f\left(g h, h^{-1}\right) .
$$


The statement $f\left(\sigma h, h^{-1} \sigma^{-1} \tau k\right) \in W_{i}^{\times}$is then a consequence of the following identities:

$$
\begin{aligned}
f^{\sigma}\left(h, h^{-1} \sigma^{-1} \tau k\right) f\left(\sigma, \sigma^{-1} \tau k\right) & =f(\sigma, h) f\left(\sigma h, h^{-1} \sigma^{-1} \tau k\right), \\
f^{\sigma}\left(\sigma^{-1} \tau, k\right) f\left(\sigma, \sigma^{-1} \tau k\right) & =f\left(\sigma, \sigma^{-1} \tau\right) f(\tau, k) .
\end{aligned}
$$

The fact that the relation is a partial ordering and satisfies part (b) is now a consequence of the following:

If $\sigma, \tau, \gamma \in H_{i-1}$ then

$$
f^{\sigma}\left(\sigma^{-1} \tau, \tau^{-1} \gamma\right) f\left(\sigma, \sigma^{-1} \gamma\right)=f\left(\sigma, \sigma^{-1} \tau\right) f\left(\tau, \tau^{-1} \gamma\right)
$$

We begin our characterization of those standard orders which are valuation rings in the case where $|Y|=1$, that is $f(G \times G) \subseteq W$ and $B_{f}=\sum_{\sigma \in G} W x_{\sigma}$. The first result shows that this condition is quite restrictive.

Proposition 2.10. Let $V$ be a valuation ring in $F$ and let $K$ be an inertial Galois extension with Galois group $G$. Let $W$ be the unique extension of $V$ to $K$. Let $f \in Z^{2}\left(G, K^{\times}\right)$be a normalized two cocycle and assume $f$ is standard for $Y=\{V\}$. If $B_{f}=\sum_{\sigma \in G} W x_{\sigma}$ is a valuation ring then every proper overring of $B_{f}$ is Azumaya.

Proof. If $C$ is a proper overring of $B_{f}$ then $V^{\prime}=Z(C)$ is a proper overring of $V$ and $W^{\prime}=W V^{\prime}$ is a proper overring of $W$. Moreover $C=B_{f} V^{\prime}$ and $C \cap K=W^{\prime}$. In particular $C$ is the standard order corresponding to $f$ viewed as standard for $Y^{\prime}=\left\{V^{\prime}\right\}$. Let $H^{\prime}=\left\{\sigma \in G \mid f\left(\sigma, \sigma^{-1}\right) \in W^{\prime \times}\right\}$. By Proposition 2.6 we know

$$
J(C)=\sum_{\sigma \in H^{\prime}} J\left(W^{\prime}\right) x_{\sigma}+\sum_{\sigma \notin H^{\prime}} W^{\prime} x_{\sigma} .
$$

But $J(C) \subseteq B_{f}$. Because $W^{\prime}$ is a proper overring of $W$ we infer that $H^{\prime}=G$ and so $C$ is Azumaya.

To continue the characterization we first prove a more general result. Let $f$ be standard for $Y=\left\{V_{1}, V_{2}, \ldots, V_{n-1}, V_{n}\right\}$. Let $w$ denote the value function determined by $f$ and let $B_{w}=\sum_{\sigma \in G} N_{\sigma} x_{\sigma}$ denote the corresponding valuation ring. Recall that $N_{\sigma}=\left\{k \in K \mid w\left(k x_{\sigma}\right) \geq 0\right\}$. It is shown in the proof of [MW, Theorem 2.1] that $w\left(x_{\sigma} s\right)=w\left(x_{\sigma}\right)+w(s)$ for all $\sigma \in G$ and $s \in \Sigma_{f}$. Moreover letting $\Gamma_{w}=w\left(\Sigma_{f}\right)$, the map $\alpha: G \rightarrow \Gamma_{w} / \Gamma$ given by $\alpha(\sigma)=w\left(x_{\sigma}\right)+\Gamma$ is a surjective homomorphism. We let $I$ denote the kernel of $\alpha$, so $I=\{\sigma \in$ $\left.G \mid w\left(x_{\sigma}\right) \in \Gamma\right\}$.

Lemma 2.11. Let $B_{w}=\sum_{\sigma \in G} N_{\sigma} x_{\sigma}$ be the valuation ring of $w$ and assume each $N_{\sigma}$ is a ring. Then:

(a) For all $\sigma \in G, f\left(\sigma, \sigma^{-1}\right) \in W$.

(b) We have $I=\left\{\sigma \in G \mid f\left(\sigma, \sigma^{-1}\right) \in W^{\times}\right\}$.

Proof. Let $B=B_{w}$. First observe that for each $\sigma$, if $N_{\sigma}$ is a ring then $1 \in N_{\sigma}$ and so $W=N_{\text {id }} \subseteq N_{\sigma}$.

(a) Because $1 \in N_{\sigma}$ for all $\sigma$, we have $x_{\sigma} \in B$ for all $\sigma$, and so $W$ contains $x_{\sigma} x_{\sigma^{-1}}=f\left(\sigma, \sigma^{-1}\right)$.

(b) Because $x_{\tau} \in B$ for all $\tau \in G, w\left(x_{\tau}\right) \geq 0$. Let $H=\left\{\sigma \in G \mid f\left(\sigma, \sigma^{-1}\right) \in\right.$ $\left.W^{\times}\right\}$. If $\sigma \in H$, then

$$
0=v\left(f\left(\sigma, \sigma^{-1}\right)\right)=w\left(x_{\sigma} x_{\sigma^{-1}}\right)=w\left(x_{\sigma}\right)+w\left(x_{\sigma^{-1}}\right) .
$$


It follows that $w\left(x_{\sigma}\right)=0$, so $\sigma \in I$. Conversely if $\sigma \in I$, then $w\left(x_{\sigma}\right) \in \Gamma$, say $w\left(x_{\sigma}\right)=v(a), a \in K$. Note that $v(a) \geq 0$. Because

$$
W_{\sigma}=\left\{k \in K \mid w\left(k x_{\sigma}\right) \geq 0\right\}=\left\{k \in K \mid w(k) \geq-w\left(x_{\sigma}\right)\right\}
$$

it follows that $W_{\sigma}=a^{-1} W$. But $W_{\sigma}$ and $W$ are both rings. Hence $a$ is a unit in $W$, so $w\left(x_{\sigma}\right)=v(a)=0$.

Now assume $f$ is standard for $\{V\}$, so $B_{f}=\sum_{\sigma \in G} W x_{\sigma}$.

Lemma 2.12. (a) We have $B_{f} \subseteq B_{w}$. In particular if $B_{f}$ is a valuation ring, then $B_{f}=B_{w}$.

(b) There is a cocycle $g$ equivalent to $f$ such that $B_{w}=B_{g}$ if and only if $B_{w}$ is finitely generated as a $V$-module.

Proof. (a) The ring $B_{f}$ is finitely generated as a $V$-module, because $W$ is a finitely generated $V$-module. In particular each $x_{\sigma}$ is integral over $V$. If $k$ is the order of $\sigma$, then $x_{\sigma}^{k}=f(\sigma, \sigma) f\left(\sigma^{2}, \sigma\right) \cdots f\left(\sigma^{k-1}, \sigma\right)$ is integral over $V$ and lies in $K$. Thus $x_{\sigma}^{k} \in W$ and so $0 \leq w\left(x_{\sigma}^{k}\right)=k w\left(x_{\sigma}\right)$. Hence $x_{\sigma} \in B_{u}$. This means $1 \in N_{\sigma}$ and thus $W \subseteq N_{\sigma}$ because $N_{\sigma}$ is a $W$-module. Therefore $B_{f} \subseteq B_{w}$. If $B_{f}$ is a valuation ring, then $B_{f}=B_{w}$ because $B_{f} \cap F=B_{w} \cap F$.

(b) If $B_{w}=B_{g}$ then in particular $B_{w}$ is finitely generated over $V$. Conversely, assume $B_{w}$ is finitely generated. It follows that for each $\sigma$ the $V$ submodule $N_{\sigma}$ is finitely generated. Because $N_{\sigma} N_{\sigma^{-1}}^{\sigma} f\left(\sigma, \sigma^{-1}\right) \subseteq N_{\mathrm{id}}=W$, each $N_{\sigma}$ is also a fractional ideal, and so we conclude that $N_{\sigma}$ is principal over $W$. It follows that there is a cocycle $g$ equivalent to $f$ such that $B=B_{g}$.

It is shown in [M, Proposition 3.2] that if $w$ is any value function then $B_{w}$ is a finitely generated $V$-module if and only if $\left[\Sigma_{f}: F\right]=\varepsilon f$, where $f=\left[\bar{B}_{w}: \bar{V}\right]$ and $\varepsilon$ is the number of elements in the set $\Lambda=\left\{\gamma \in \Gamma_{w} \mid 0 \leq \gamma<\delta\right.$ for all $\left.\delta \in \Gamma^{+}\right\}$. Moreover in this case $\varepsilon=\left[\Gamma_{w}: \Gamma\right]$ and $\Gamma_{w} / \Gamma$ is a cyclic group generated by $\gamma_{0}+\Gamma$, where $\gamma_{0}$ is the least positive element of $\Gamma_{w}$. We want to give another characterization of when $B_{f}$ is a valuation ring.

Lemma 2.13. Set $\Lambda=\left\{\gamma \in \Gamma_{w} \mid 0 \leq \gamma<\delta\right.$ for all $\left.\delta \in \Gamma^{+}\right\}$. Then $B_{f}$ is a valuation ring if and only if $w\left(x_{\sigma}\right) \in \Lambda$ for all $\sigma \in G$.

Proof. Suppose $B_{f}$ is a valuation ring. We have seen that it follows that $B_{f}=$ $B_{w}$. Let $a$ be an element of $K$ such that $0<v(a)<w\left(x_{\sigma}\right)$ for some $\sigma$ in $G$. Then $w\left(a^{-1} x_{\sigma}\right) \geq 0$ so $a^{-1} x_{\sigma} \in B_{w}=B_{f}$. Thus $a^{-1} \in W$, so $v(a) \leq 0$. This is a contradiction, so $w\left(x_{\sigma}\right) \in \Lambda$.

Conversely, suppose $w\left(x_{\sigma}\right) \in \Lambda$ for all $\sigma \in G$. If $\sum_{\sigma \in G} a_{\sigma} x_{\sigma} \in B_{w}$ then $v\left(a_{\sigma}\right)+w\left(x_{\sigma}\right) \geq 0$ for all $\sigma$. If $v\left(a_{\sigma}\right)<0$ for some $\sigma$ then $0<$ $-v\left(a_{\sigma}\right) \leq w\left(x_{\sigma}\right)$, contradicting $w\left(x_{\sigma}\right) \in \Lambda$. Thus $a_{\sigma} \in W$ for all $\sigma$, so $B_{w} \subseteq \sum_{\sigma \in G} W x_{\sigma}=B_{f}$. But $B_{f} \cap F=V$, so $B_{w}=B_{f}$.

Let $H=\left\{\sigma \in G \mid f\left(\sigma, \sigma^{-1}\right) \in W^{\times}\right\}$. We can now characterize those cocycles $f$ for which $B_{f}=\sum_{\sigma \in G} W x_{\sigma}$ is a valuation ring. The characterization generalizes Theorem 2.3 of $[\mathrm{H}]$.

Theorem 2.14. Suppose $K / F$ is Galois and inertial with respect to the valuation rings $W / V$. Let $f \in Z^{2}\left(G, K^{\times}\right)$be a normalized cocycle with $f(G \times G) \subseteq$ $W$. The ring $B_{f}=\sum_{\sigma \in G} W x_{\sigma}$ is a valuation ring if and only if the following conditions are satisfied:

(a) $H$ is normal in $G$ and the quotient $G / H$ is cyclic. 
(b) Either $H=G$, in which case $B_{f}$ is Azumaya, or there is an element $\sigma$ in $G$ such that $\sigma H$ generates $G / H$ and such that the partial ordering on $G / H$ is the simple chain $H \leq \sigma H \leq \sigma^{2} H \leq \sigma^{3} H \leq \cdots \leq \sigma^{k-1} H$ where $k$ is the order of $\sigma$. Moreover $f\left(\sigma, \sigma^{-1}\right) \in J(W)-J(W)^{2}$.

Proof. Assume $B_{f}$ is a valuation ring. Then we know $B_{f}=B_{w}$, the valuation ring of the value function $w$ determined by $f$. By Lemma 2.13, $w\left(x_{\sigma}\right) \in \Lambda$ for all $\sigma \in G$. Also $|\Lambda|=\left|\Gamma_{w} / \Gamma\right|$ and $\Gamma_{w} / \Gamma$ is cyclic. By Lemma 2.11, $H=I=\operatorname{ker}(\alpha)$. Hence $H$ is a normal subgroup of $G$, and because the map $\alpha: G \rightarrow \Gamma_{w} / \Gamma$ is surjective, $G / H \cong \Gamma_{w} / \Gamma$ is cyclic. If $H=G$ then $f(G \times G) \subseteq W^{\times}$and so $B_{f}$ is Azumaya. Assume therefore that $H \neq G$. Let $\gamma$ be the least positive element of $\Gamma_{w}$ and let $\sigma$ be an element of $G$ such that $w\left(x_{\sigma}\right)=\gamma$. Then $\alpha(\sigma)=\gamma+\Gamma$ and $\langle\gamma+\Gamma\rangle=\Gamma_{w} / \Gamma$, so $\langle\sigma H\rangle=G / H$. Hence the order of $\sigma H$ is $\varepsilon=|\Lambda|$. Because

$$
x_{\sigma}^{\varepsilon-1}=\left(\prod_{i=1}^{\varepsilon-2} f\left(\sigma^{i}, \sigma\right)\right) x_{\sigma^{\varepsilon-1}}
$$

we have

$$
(\varepsilon-1) \gamma=v\left(\prod_{i=1}^{\varepsilon-2} f\left(\sigma^{i}, \sigma\right)\right)+w\left(x_{\sigma^{\varepsilon-1}}\right) .
$$

Hence $(\varepsilon-1) \gamma \geq v\left(\prod_{i=1}^{\varepsilon-2} f\left(\sigma^{i}, \sigma\right)\right)$. But it is easy to see that $(\varepsilon-1) \gamma \in \Lambda$. Hence $v\left(\prod_{i=1}^{\varepsilon-2} f\left(\sigma^{i}, \sigma\right)\right)=0$, so $f\left(\sigma^{i}, \sigma\right) \in W^{\times}$for $0 \leq i \leq \varepsilon-1$. It follows that $\sigma^{i} H \leq \sigma^{i+1} H$ for $0 \leq i \leq \varepsilon-1$. Moreover $x_{\sigma}^{\varepsilon}=\left(\prod_{i=1}^{\varepsilon-1} f\left(\sigma^{i}, \sigma\right)\right)$, so $\varepsilon \gamma=w\left(x_{\sigma}^{\varepsilon}\right)=v\left(f\left(\sigma^{\varepsilon-1}, \sigma\right)\right)$. Because $\gamma$ is the least positive element of $\Gamma_{w}$ it follows that $\varepsilon \gamma$ is the least positive element of $\Gamma$. Hence $f\left(\sigma^{\varepsilon-1}, \sigma\right) \in J(W)-$ $J(W)^{2}$. We will have finished this direction once we show that $v\left(f\left(\sigma^{\varepsilon-1}, \sigma\right)\right)=$ $v\left(f\left(\sigma, \sigma^{-1}\right)\right)$. But $\sigma^{\varepsilon-1}=h \sigma^{-1}$ for some $h \in H$ and $f(H \times G) \subseteq W^{\times}$. From the cocycle identity $f^{h}\left(\sigma^{-1}, \sigma\right)=f\left(h, \sigma^{-1}\right) f\left(h \sigma^{-1}, \sigma\right)$ we infer

$$
\begin{aligned}
v\left(f\left(\sigma^{\varepsilon-1}, \sigma\right)\right) & =v\left(f\left(h \sigma^{-1}, \sigma\right)\right)=v\left(f^{h}\left(\sigma^{-1}, \sigma\right)\right) \\
& =v\left(f\left(\sigma^{-1}, \sigma\right)\right)=v\left(f\left(\sigma, \sigma^{-1}\right)\right)
\end{aligned}
$$

where the last equality follows from $f^{\sigma}\left(\sigma^{-1}, \sigma\right)=f\left(\sigma, \sigma^{-1}\right)$.

For the converse suppose $H$ is normal in $G$. If $H=G$ then $B_{f}$ is Azumaya. Otherwise we have $G / H=\langle\sigma H\rangle$ with $f\left(\sigma, \sigma^{-1}\right) \in J(W)-J(W)^{2}$ and $\sigma^{i} H<$ $\sigma^{i+1} H$ for $0 \leq i<|G / H|$. We will be done by Lemma 2.13 if we show $w\left(x_{\sigma}\right) \in \Lambda$ for all $\sigma \in G$. Let $t=|G / H|$. The relation $\sigma^{i} H<\sigma^{i+1} H$ gives $f\left(\sigma^{i}, \sigma\right) \in W^{\times}$for $0 \leq i<t-1$ so $w\left(x_{\sigma^{i}}\right)=i w\left(x_{\sigma}\right)$ for $i<t$ and $w\left(x_{\sigma}^{t}\right)=v\left(f\left(\sigma^{t-1}, \sigma\right)\right)$. Let $\gamma=w\left(x_{\sigma}\right)$. If $\tau \in G$, say $\tau=\sigma^{i} h$, then

$$
w\left(x_{\tau}\right)=w\left(x_{\sigma^{i}}\right)+w\left(x_{h}\right)-v\left(f\left(\sigma^{i}, h\right)\right)=w\left(x_{\sigma^{i}}\right)=i \gamma
$$

because $h \in H=I$ and $f\left(\sigma^{i}, h\right) \in W^{\times}$. As in the first half of the proof we have $v\left(f\left(\sigma^{t-1}, \sigma\right)\right)=v\left(f\left(\sigma, \sigma^{-1}\right)\right)$. Because $f\left(\sigma, \sigma^{-1}\right) \in J(W)-J(W)^{2}$, we obtain $v\left(f\left(\sigma^{t-1}, \sigma\right)\right)=w\left(x_{\sigma}^{t}\right)=t \gamma$ is the least positive element of $\Gamma$. Thus $w\left(x_{\tau}\right)=i \gamma<t \gamma$ for all $\tau$, so $w\left(x_{\tau}\right) \in \Lambda$. Thus $B_{f}=B_{w}$.

Recall that from Lemma 2.11 we know $H=I=\operatorname{ker} \alpha$. In particular $H$ can be described using the value function $w$. It is worth noting that under the 
hypotheses of Theorem 2.14 the partial ordering on $G / H$ (which is then a total ordering) can also be described in terms of $w$. In fact $\sigma H \leq \tau H$ if and only if $w(\sigma) \leq w(\tau)$ : If $\sigma H \leq \tau H$ then $f\left(\sigma, \sigma^{-1} \tau\right) \in W^{\times}$, so $w\left(f\left(\sigma, \sigma^{-1} \tau\right)\right)=0$. Hence from $x_{\sigma} x_{\sigma^{-1} \tau}=f\left(\sigma, \sigma^{-1} \tau\right) x_{\tau}$, we infer that $w\left(x_{\sigma}\right)+w\left(x_{\sigma^{-1} \tau}\right)=w\left(x_{\tau}\right)$ and so that $w\left(x_{\sigma}\right) \leq w\left(x_{\tau}\right)$. (This direction is true in general, that is even if the ordering on $G / H$ is not total.) For the converse suppose $w(\sigma) \leq w(\tau)$. If $\tau H<\sigma H$ then by the first part $w(\tau) \leq w(\sigma)$ and so $w(\tau)=w(\sigma)$. But then $\sigma^{-1} \tau \in \operatorname{ker} \alpha=H$, a contradiction. Because $G / H$ is totally ordered it follows that $\sigma H \leq \tau H$.

Here is a nice application of the theorem. The result can also be obtained using the exact sequence (5.4) of [JW] along with [W, Theorems B, F].

Corollary 2.15. Assume the hypotheses of the proposition and assume that the value group of $V$ is equal to its own divisible hull. If $f \in Z^{2}\left(G, K^{\times}\right)$is a normalized two cocycle and $B$ is a valuation ring over $V$ in $\Sigma_{f}$ then $B$ is Azumaya.

Proof. Let $B$ be a valuation ring over $V$ in $\Sigma_{f}$. The condition on the value group implies that the ramification index of $B / V$ is one. Moreover by [MW, Theorem 2.1] $B / V$ is defectless. It then follows from [M, Proposition 3.2] that $B$ is finitely generated as a $V$-module. By Lemma 2.12 it follows we may assume $f$ is standard for $\{V\}$ and $B=B_{f}=\sum_{\sigma \in G} W x_{\sigma}$. Let $H=\{\sigma \in$ $\left.G \mid f\left(\sigma, \sigma^{-1}\right) \in W^{\times}\right\}$. If $H$ is a proper subgroup of $G$ then by the theorem there is an element $\sigma \in G-H$ such that $f\left(\sigma, \sigma^{-1}\right) \in J(W)-J(W)^{2}$. But because the valuation is not discrete $J(W)=J(W)^{2}$, so this is impossible. Hence $H=G$, so $B$ is Azumaya.

We now proceed to the general case. We begin with a generalization of Lemma 2.12 .

Lemma 2.16. Let $Y=\left\{V_{1}, V_{2}, \ldots, V_{n-1}, V_{n}\right\}$ where $V=V_{n} \subseteq V_{n-1} \subseteq \cdots \subseteq$ $V_{1} \subseteq V_{0}=F$. There is a cocycle $g$ equivalent to $f$ such that $g$ is standard for $Y$ and $B_{w}=B_{g}$ if and only if for all $i, 1 \leq i \leq n-1, B_{w} V_{i} / J\left(B_{w} V_{i-1}\right)$ is finitely generated over $V_{i} / J\left(V_{i-1}\right)$.

Proof. If $B_{w}=B_{g}$ then we can apply Proposition 2.6 to see that for all $i$, $1 \leq i \leq n-1$,

$$
B_{w} V_{i} / J\left(B_{w} V_{i-1}\right)=\sum_{\sigma \in H_{i}} W_{i} / J\left(W_{i}\right) x_{\sigma}
$$

which is finitely generated over $V_{i} / J\left(V_{i-1}\right)$.

For the converse we proceed by induction on $n$. Let $B_{w}=\sum_{\sigma \in G} N_{\sigma} x_{\sigma}$ as usual. If $n=1$ then this is the second part of Lemma 2.12. Assume $n>1$. The induction hypothesis applied to $Y^{\prime}=Y-\{V\}$ shows that there is a cocycle $g^{\prime}$ equivalent to $f$ such that the valuation ring $B_{w} V_{n-1}$ equals $B_{g^{\prime}}$. Let $B_{g^{\prime}}=\sum_{\sigma \in G} W_{\sigma} y_{\sigma}$. By Proposition 2.6 we know

$$
J\left(B_{g^{\prime}}\right)=\sum_{\sigma \in H_{n-1}} J\left(W_{n-1}\right) y_{\sigma}+\sum_{\sigma \notin H_{n-1}} W_{\sigma} y_{\sigma} .
$$

Because $J\left(B_{g^{\prime}}\right) \subseteq B_{w}$ we can write

$$
B_{w}=\sum_{\sigma \in H_{n-1}} N_{\sigma} y_{\sigma}+\sum_{\sigma \notin H_{n-1}} W_{\sigma} y_{\sigma}
$$


where $N_{\text {id }}=W$ and $N_{\sigma} \subseteq W_{n-1}$ with $N_{\sigma} W_{n-1}=W_{n-1}$ for all $\sigma \in H_{n-1}$. By hypothesis

$$
B_{w} / J\left(B_{g^{\prime}}\right)=\sum_{\sigma \in H_{n-1}} N_{\sigma} / J\left(W_{n-1}\right) y_{\sigma}
$$

is a finitely generated $V / J\left(V_{n-1}\right)$-module and clearly contains $W / J\left(W_{n-1}\right)$. As in the proof of part (b) of Lemma 2.12 it follows that for each $\sigma \in H_{n-1}$ there is an element $a_{\sigma} \in W_{n-1}$ such that $N_{\sigma} / J\left(W_{n-1}\right)$ is the principal ideal $W / J\left(W_{n-1}\right)\left(a_{\sigma}+J\left(W_{n-1}\right)\right)$. Hence $W a_{\sigma}+J\left(W_{n-1}\right)=N_{\sigma}$. But then $W a_{\sigma} W_{n-1}$ $+J\left(W_{n-1}\right)=N_{\sigma} W_{n-1}=W_{n-1}$ so $a_{\sigma}$ is a unit in $W_{n-1}$. It follows that $J\left(W_{n-1}\right) \subseteq W a_{\sigma}$ and so $W a_{\sigma}=N_{\sigma}$. It is now easy to see that one can alter $g^{\prime}$ to obtain a cocycle $g$ equivalent to $f$ such that $g$ is standard for $Y$ and $B_{w}=B_{g}$.

Theorem 2.17. Let $f$ be a standard cocycle for $Y=\left\{V_{1}, V_{2}, \ldots, V_{n-1}, V_{n}\right\}$. The crossed product order $B_{f}$ is a valuation ring if and only if for each $i$, $1 \leq i \leq n$, the following conditions are satisfied:

(a) The subgroup $H_{i}$ is normal in $H_{i-1}$, and the quotient $H_{i-1} / H_{i}$ is cyclic.

(b) Either $H_{i-1}=H_{i}$ or there is an element $\sigma_{i-1}$ in $H_{i-1}$ such that $\sigma_{i-1} H_{i}$ generates $H_{i-1} / H_{i}$ and such that the partial ordering on $H_{i-1}$ is the simple chain

$$
\sigma_{i-1} H_{i} \leq \sigma_{i-1}^{2} H_{i} \leq \sigma_{i-1}^{3} H_{i} \leq \cdots \leq \sigma_{i-1}^{k-1} H_{i}
$$

where $k$ is the order of $\sigma_{i-1}$. Moreover $f\left(\sigma_{i-1}, \sigma_{i-1}^{-1}\right) \in J\left(W_{i}\right)-J\left(W_{i}\right)^{2}$.

Proof. Let $B=B_{f}$ and assume $B$ is a valuation ring. We proceed by induction on $n$. We need to show that for each $i, f$ satisfies properties (a) and (b). If $n=1$ then this is Theorem 2.14. Hence we may assume $n>1$. The ring $B_{n-1}=B V_{n-1}$ is also a valuation ring. Moreover we have seen that $B_{n-1}$ is the crossed product over $V_{n-1}$ corresponding to the cocycle $f$, which is standard for $Y^{\prime}=Y-\{V\}$. Hence by induction properties (a) and (b) hold for all $i$, $1 \leq i \leq n-1$, and we are left with verifying the properties for $i=n$. Let $H=H_{n-1}$. We have $J\left(B_{n-1}\right) \subseteq B$ by Proposition 2.6 and $B / J\left(B_{n-1}\right)$ is a valuation ring in the simple algebra $B_{n-1} / J\left(B_{n-1}\right)$. Also

$$
B_{n-1} / J\left(B_{n-1}\right)=\sum_{\sigma \in H} W_{n-1} / J\left(W_{n-1}\right) x_{\sigma},
$$

a crossed product algebra for the cocycle $f_{\mid H \times H}$. Moreover $f_{\mid H \times H}$ is standard for the valuation ring $W_{n} / J\left(W_{n-1}\right)$ and

$$
B / J\left(B_{n-1}\right)=\sum_{\sigma \in H} W_{n} / J\left(W_{n-1}\right) x_{\sigma}
$$

is the crossed product order for $f_{\mid H \times H}$. Hence the result for $i=n$ follows from the $n=1$ case.

Conversely assume $B=B_{f}$ and $f$ has properties (a) and (b). We need to show $B$ is a valuation ring. Again we argue by induction on $n$, the $n=1$ case being Theorem 2.14. Thus assume $n>1$ and let $B_{n-1}=B V_{n-1}$. Then $B_{n-1}$ is the crossed product order for the standard cocycle $f$ (for $Y^{\prime}$ ). Because $f$ satisfies (a) and (b) for $i \leq n-1$ we infer by induction that $B_{n-1}$ is a valuation ring. Moreover $J\left(B_{n-1}\right) \subseteq B$ and as we have seen $B / J\left(B_{n-1}\right)$ is the 
crossed product order for the cocycle $f_{\mid H \times H}$ inside the crossed product algebra $B_{n-1} / J\left(B_{n-1}\right)$ (where $\left.H=H_{n-1}\right)$. Because $f_{\mid H \times H}$ satisfies (a) and (b) for $H_{n} \subseteq H$ we obtain $B / J\left(B_{n-1}\right)$ is a valuation ring by the $n=1$ case. But by [ $\mathrm{D}_{2}, \S 1$, Proposition 2] it then follows that $B$ is a valuation ring, as desired.

Example 2.18. Here is an example of a valuation ring which is not equal to $B_{f}$ for any $f$ and $Y$ :

Let $F$ be a field with valuation $v$ whose value group is $\Gamma=\mathbb{Z}+\pi \mathbb{Z}$ with the archimedian ordering induced from the inclusion $\Gamma \subseteq \mathbb{R}$. Let $V$ be the valuation ring of $v$ and suppose $\operatorname{char}(\bar{V}) \neq 2$ and that there is an $a \in V$ with $\bar{a}$ not a square in $\bar{V}$. (For example we could take $F=\mathbb{Q}(\Gamma)$ and $a=2$.) Let $b \in V$ with $v(b)=\pi$. Set $K=F(\sqrt{a})$, an inertial Galois extension of $F$ with respect to $V$ and $\Sigma$ the cyclic algebra $(K / F, \sigma, b)$. Then $\Sigma$ is the quaternion algebra $(a, b)_{F}$ with generators $i, j$ satisfying $i^{2}=a, j^{2}=b, i j=-j i$. Also $K=F(i)$ and $\Sigma=K \oplus K j$. By [JW, Example 4.3] $v$ extends to a valuation on $\Sigma$, which we will also denote by $v$, such that $v(\alpha+\beta j)=\min \{v(\alpha), v(\beta)+\pi / 2\}$ for $\alpha, \beta \in K$. Let $B$ be the valuation ring of this valuation. Then $B=W \oplus T j$ where $W=B \cap K$ and $T=\{\alpha \in K \mid v(\alpha) \geq-\pi / 2\}$. If $B=B_{f}$ for some $f$ then $B=W \oplus W x_{\sigma}$ is a finitely generated $V$-module. Thus $T$ is a finitely generated $W$-module, hence principal. But if $T=W x$ then $v(x)$ is the least element of $v(T)=\{\gamma \in \Gamma \mid v(\gamma) \geq-\pi / 2\}$. But $v(T)$ has no least element because $-\pi / 2 \notin \Gamma$ and $\Gamma$ is dense in $\mathbb{R}$. Hence $B$ is not a crossed product order $B_{f}$ for any $f$.

We end this section with a proposition that will be useful in the next section.

Proposition 2.19. Suppose $f$ is standard for $W_{n}$. Let $w$ be the value function associated to $f$ and assume that $B_{w}=\sum_{\sigma \in G} W_{\sigma} x_{\sigma}$ is in standard form (that is $B_{w}=B_{f}$ ). If $H$ is any subgroup of $G$, then $\sum_{\sigma \in H} W_{\sigma} x_{\sigma}$ is a valuation ring in $\sum_{\sigma \in H} K x_{\sigma}$.

Proof. Let $E=\sum_{\sigma \in H} K x_{\sigma}$ and let $w^{\prime}=\left.w\right|_{E}$. Then $C=B_{w} \cap E=\{z \in$ $\left.E \mid w^{\prime}(z) \geq 0\right\}$. Let $J=\left\{z \in E \mid w^{\prime}(z)>0\right\}$. By [M, Theorem 2.4] we will be done if we can show $w^{\prime}$ is a value function and $C / J$ is simple. Moreover to show $w^{\prime}$ is a value function, it suffices to show that if $y \in \operatorname{im}\left(w^{\prime}\right)$, then there exists $z \in E^{\times}$such that $w^{\prime}(z)=y$ and $w^{\prime}\left(z^{-1}\right)=-w^{\prime}(z)$. But the definition of $w$ shows that $\operatorname{im}\left(w^{\prime}\right)=\left\{w(a)+w\left(x_{\sigma}\right) \mid a \in F, \sigma \in H\right\}$. Because $w(a)+w\left(x_{\sigma}\right)=w\left(a x_{\sigma}\right)$ and $w\left(\left(a x_{\sigma}\right)^{-1}\right)=-w\left(a x_{\sigma}\right)$, we have shown $w^{\prime}$ is a value function.

We now proceed to show $C / J$ is simple. Let $g=f_{\mid H \times H}$ and let $H_{i}^{\prime}=$ $H \cap H_{i}=\left\{\sigma \in H \mid f\left(\sigma, \sigma^{-1}\right) \in W_{i}^{\times}\right\}$for $0 \leq i \leq n$. Then for $i<n$, $\left(H_{i}^{\prime} \times H_{i}^{\prime}\right) \subseteq f\left(H_{i} \times H_{i}\right) \subseteq W_{i+1}$, so $g$ is standard for $W_{n}$ and $C=B_{g}$. By Proposition 2.6 $C$ is primary and

$$
J(C)=\sum_{\sigma \in H_{n}^{\prime}} J\left(W_{\sigma}\right) x_{\sigma}+\sum_{\sigma \notin H_{n}^{\prime}} W_{\sigma} x_{\sigma} .
$$

But then $J=J(B) \cap C=J(C)$ so $C / J$ is simple.

\section{DisCRETE RANK $N$ VALUATION RINGS}

In this section we consider the case where $V$ is unramified but not necessarily indecomposed in $K$. We restrict our attention to discrete rank $n$ valuation 
rings $V$. Let $V=V_{n} \subseteq V_{n-1} \subseteq \cdots \subseteq V_{2} \subseteq V_{1} \subseteq F$ be the overrings of $V$ and let $W_{i}$ be the integral closure of $V_{i}$ in $K$.

Let $f \in Z^{2}\left(G, K^{\times}\right)$be a normalized two-cocycle and let $(K / F, G, f)=$ $\sum_{\sigma \in G} K x_{\sigma}$. By Proposition 1.7 we may assume there is a valuation ring $B=$ $\sum_{\sigma \in G} W_{\sigma} x_{\sigma}$ over $V$ in $(K / F, G, f)$, where each $W_{\sigma}$ is an overring of $W_{\text {id }}$.

In order to reduce the confusion caused by the too frequent use of the letter $W$ in our notation, we will let $S=W_{1}$. That is $S$ will denote the integral closure of $V$ in $K$.

We want to determine the rings $W_{\sigma}$. Recall from $\S 1$ that each $W_{\sigma}$ is a semilocal Prüfer ring and hence an intersection of valuation rings. More specifically $W_{\sigma}=\bigcap S_{P}$ where the intersection is over those prime ideals $P$ of $S$ such that $W_{\sigma} \subseteq S_{P}$. Also note that $V=B \cap F=W_{\text {id }} \cap F$.

Definition. A prime $Q$ of $S$ is said to belong to $B$ if $W_{\text {id }} \subseteq S_{Q}$.

Observe that for every $i \leq n$ there is a prime ideal of height $i$ belonging to $B$ : It suffices to show there is a maximal ideal $M$ of $S$ belonging to $B$ because then any prime ideal contained in $M$ also belongs to $B$. But if no maximal ideal belongs to $B$ then $W_{\text {id }} \supseteq W_{2}$ and so $W_{\text {id }} \cap F=V_{2}$, a contradiction.

The following is the basic result of this section.

Theorem 3.1. Let $B=\sum_{\sigma \in G} W_{\sigma} x_{\sigma}$ be a valuation ring over $V$. Then

(a) We have $J(B)=\sum_{\sigma \in G} J_{\sigma} x_{\sigma}$ where for each $\sigma \in G, J_{\sigma}=\{k \in$ $\left.W_{\sigma} \mid k f\left(\sigma, \sigma^{-1}\right) \in J\left(W_{\sigma}\right)\right\}$.

(b) If $Q_{1}, Q_{2}$ are prime ideals of $S$ of the same height belonging to $B$, then there exists $\sigma \in G$ such that $Q_{1}^{\sigma}=Q_{2}$ and $f\left(\sigma^{-1}, \sigma\right) \notin Q_{1} W_{\text {id }}$.

Proof. The proof is by induction on the rank. If the rank is one then the theorem is a consequence of [H, Proposition 3.1, Theorem 3.2]. Hence we may assume the rank $n$ is greater than one. Because $B_{n-1}=B V_{n-1}$ is a valuation ring of rank $n-1$ the results may be assumed true for it. We will assume the theorem for valuation rings of rank less than $n$ in the following lemmas.

If $P$ is a prime ideal of $S$ of height $i<n$ we will let $S^{P}$ denote $\cap S_{Q}$ where the intersection is over those primes $Q$ of $S$ that contain $P$. Note that if $U$ is any overring of $S$ then $U S^{P}=\bigcap S_{Q}$ where this intersection is over those primes $Q$ such that $P \subseteq Q$ and $U \subseteq S_{Q}$.

Lemma 3.2. Let $P$ be a prime of height $i$ belonging to $B$. Let $\sigma \in G$.

(a) If $f\left(\sigma, \sigma^{-1}\right) \notin P W_{\text {id }}$ then $W_{\sigma} S^{P}=W_{\mathrm{id}} S^{P}$.

(b) If $Q \supseteq P$ is a prime of height $i+1$ belonging to $B$ and $f\left(\sigma, \sigma^{-1}\right) \notin$ $P W_{\mathrm{id}}$, then $W_{\mathrm{id}} \subseteq S_{Q^{\sigma^{-1}}}$ and $W_{\sigma^{-1}}^{\sigma} \subseteq S_{Q}$.

(c) If $f\left(\sigma, \sigma^{-1}\right) \in P W_{\text {id }}$, then $W_{\sigma} S^{P}=W_{\sigma} S_{P}$.

Proof. (a) Let $Q$ be a height $i+1$ prime of $S$ containing $P$. We need to show that $W_{\text {id }} \subseteq S_{Q}$ if and only if $W_{\sigma} \subseteq S_{Q}$. We know $W_{\text {id }} \subseteq W_{\sigma}$. Hence certainly $W_{\sigma} \subseteq S_{Q}$ implies $W_{\text {id }} \subseteq S_{Q}$.

Now suppose $W_{\text {id }} \subseteq S_{Q}$, but $W_{\sigma}$ is not contained in $S_{Q}$. Then $W_{\sigma} S_{Q}$ is a ring properly containing $S_{Q}$. Hence $W_{\sigma} S_{Q}$ is valuation ring. But $P$ is the unique prime of height $n$ contained in $Q$. It follows that $W_{\sigma} S_{Q} \supseteq S_{P}$. On the other hand we know $W_{\sigma} f\left(\sigma, \sigma^{-1}\right) \subseteq W_{\text {id }}$, so we have $W_{\sigma} f\left(\sigma, \sigma^{-1}\right) S_{Q} \subseteq$ 
$W_{\text {id }} S_{Q}=S_{Q}$. Thus

$$
S_{Q} \supseteq W_{\sigma} f\left(\sigma, \sigma^{-1}\right) S_{Q}=f\left(\sigma, \sigma^{-1}\right) W_{\sigma} S_{Q} \supseteq f\left(\sigma, \sigma^{-1}\right) S_{P}=S_{P},
$$

because $f\left(\sigma, \sigma^{-1}\right) \notin P W_{\text {id }}$. Thus $S_{P} \subseteq S_{Q}$, a contradiction.

(b) By part (a) $W_{\sigma} S^{P}=W_{\text {id }} S^{P}$. Hence $W_{\sigma} \subseteq S_{Q}$. But because the product $x_{\sigma} W_{\text {id }}$ is in $B$ we see that $W_{\text {id }}^{\sigma} \subseteq W_{\sigma}$. Hence $W_{\text {id }} \subseteq S_{Q^{\sigma^{-1}}}$. In particular $W_{\text {id }} \subseteq S_{P^{-1}}$, so $P^{\sigma^{-1}}$ belongs to $B$. Moreover $f\left(\sigma^{-1}, \sigma\right)=f^{\sigma^{-1}}\left(\sigma, \sigma^{-1}\right) \notin$ $P^{\sigma^{-1}} W_{\text {id }}$, so by part (a) $W_{\sigma^{-1}} \subseteq S_{Q^{\sigma^{-1}}}$. Hence $W_{\sigma^{-1}}^{\sigma} \subseteq S_{Q}$.

(c) Assume $f\left(\sigma, \sigma^{-1}\right) \in P W_{\text {id }}$. Let $Q$ be a prime of height $i+1$ that contains $P$. We claim $W_{\sigma}$ is not contained in $S_{Q}$ : There are two cases: If $P W_{\sigma}=W_{\sigma}$, then from $W_{\sigma} \subseteq S_{Q}$ we obtain $W_{\sigma}=P W_{\sigma} \subseteq P S_{Q} \subseteq Q S_{Q}$, a contradiction. If $P W_{\sigma} \neq W_{\sigma}$, then $P W_{\sigma} V_{i}$ is a maximal ideal in $W_{\sigma} V_{i}$. Let $J=\left\{k \in W_{\sigma} V_{i} \mid k f\left(\sigma, \sigma^{-1}\right) \in J\left(W_{\sigma} V_{i}\right)\right\}$. We know by the induction hypothesis that $J \subseteq J\left(B_{i}\right)$. Because $B \subseteq B_{i}$ and both are valuation rings we infer that $J\left(B_{i}\right) \subseteq B$. Hence $J \subseteq W_{\sigma}$. Let $T=\prod N$ where the product is over those primes of $S$ of height at most $i$ not contained in $P$. Note that $T \subseteq J$ because $f\left(\sigma, \sigma^{-1}\right) \in P W_{\text {id }}$. Hence $W_{\sigma} V_{i} T \subseteq W_{\sigma} V_{i} J \subseteq J \subseteq W_{\sigma}$.

Now assume $W_{\sigma} \subseteq S_{Q}$. Then $W_{\sigma} V_{i} T \subseteq S_{Q}$, so $W_{\sigma} V_{i} T S_{Q} \subseteq S_{Q}$. But $T S_{Q}=S_{Q}$ : If not $T S_{Q} \subseteq Q S_{Q}$, so $T \subseteq Q$. Hence there is a prime $N$ of height at most $i$ such that $N \subseteq Q$ but $N$ is not contained in $P$. But $P \subseteq Q$, so this is not possible. Thus $T S_{Q}=S_{Q}$ and so if $W_{\sigma} V_{i} T S_{Q} \subseteq S_{Q}$, then $V_{i} \subseteq S_{Q}$, a contradiction. This proves the claim.

Because $W_{\sigma} S^{P}$ is a Prüfer ring it is the intersection of the valuation rings that contain it and each such valuation ring is a localization of $W_{\sigma} S^{P}$ at some prime ideal. We have just seen that any valuation ring that contains $W_{\sigma} S^{P}$ must have rank at most $i$. However every prime ideal of $S^{P}$ of height at most $i$ is contained in $P$ and so every valuation ring of rank at most $i$ that contains $S^{P}$ must contain $S_{P}$. Hence $W_{\sigma} S^{P} \supseteq S_{P}$ and so $W_{\sigma} S^{P}=W_{\sigma} S_{P}$.

Proposition 3.3. Let $P$ be a prime of height $i \leq n$ belonging to $B$. Let $P=$ $P_{i} \supseteq P_{i-1} \supseteq \cdots \supseteq P_{1} \supseteq P_{0}=0$ be the unique chain of prime ideals of $S$ contained in $P$. If $\sigma \in G$ then $W_{\sigma} S_{P}=S_{P_{j}}$ where $j \leq n$ is the unique integer such that $f\left(\sigma, \sigma^{-1}\right) \in P_{j} W_{\text {id }}-P_{j-1} W_{\text {id }}$.

Proof. This is an easy consequence of parts (a) and (c) of Lemma 3.2.

Proposition 3.4. We have $W_{\mathrm{id}}=\bigcap S_{M}$ where the intersection is over those maximal ideals $M$ of $S$ that belong to $B$.

Proof. It suffices to show that every prime of height $i<n$ that belongs to $B$ is contained in a height $i+1$ prime ideal belonging to $B$. By the remark immediately preceding Theorem 3.1 , we know there is some height $i+1$ prime ideal $Q$ of $S$ belonging to $B$. Let $P$ be the unique height $i$ prime of $S$ contained in $Q$. Then $P$ belongs to $B$. By the induction hypothesis applied to part (b) of the theorem, if $T$ is another height $i$ prime of $S$ belonging to $B$, then there is an element $\sigma \in G$ such that $f\left(\sigma^{-1}, \sigma\right) \notin P$ and $P^{\sigma}=T$. By part (b) of Lemma 3.2, $W_{\text {id }} \subseteq S_{Q^{\sigma}}$. But $Q^{\sigma}$ is a height $i+1$ prime ideal containing $T$, so we have proved the proposition.

It should be observed that these propositions give, for each $\sigma \in G$, a prescription for finding $W_{\sigma}$ in terms of $W_{\text {id }}$ : If $M_{1}, M_{2}, \ldots, M_{k}$ are the maximal 
ideals that belong to $B$, that is for which $W_{\text {id }} \subseteq S_{M_{i}}$, and if $M_{i}=P_{i, n} \supseteq$ $P_{i, n-1} \supseteq \cdots \supseteq P_{i, 1} \supseteq P_{i, 0}=0$ is the chain of prime ideals contained in $M_{i}$, then $W_{\sigma}=\bigcap_{i=1}^{k} S_{P_{i, j}}$ where for each $i, 1 \leq i \leq k, j_{i}$ is the unique integer such that $f\left(\sigma, \sigma^{-1}\right) \in P_{j_{i}} W_{\text {id }}-P_{j_{i}-1} W_{\text {id }}$. The description of the rings $W_{\sigma}$ is thus reduced to describing $W_{\mathrm{id}}$, or in other words to finding the primes that belong to $B$.

3.1. Proof of part (a) of the theorem. Let $\sigma \in G$. We begin by giving an alternate description of $J_{\sigma}$. We have that $J\left(W_{\sigma}\right)=\bigcap Q W_{\sigma}$ where the intersection is over those prime ideals $Q$ of $S$ such that $Q W_{\sigma}$ is a maximal ideal of $W_{\sigma}$. If $f\left(\sigma, \sigma^{-1}\right) \in Q W_{\text {id }}$ for all such $Q$, then $J_{\sigma}=W_{\sigma}$. Otherwise, that is if $f\left(\sigma, \sigma^{-1}\right) \notin J\left(W_{\sigma}\right)$, then $J_{\sigma}=\bigcap Q W_{\sigma}$ where the intersection is over those prime ideals $Q$ of $S$ such that $Q W_{\sigma}$ is a maximal ideal of $W_{\sigma}$ and $f\left(\sigma, \sigma^{-1}\right) \notin Q W_{\text {id }}$. We claim that it follows that any such $Q$ must be a maximal ideal of $S$ : Because $f\left(\sigma, \sigma^{-1}\right) \notin Q W_{\text {id }}$ we know from Lemma 3.2 that $W_{\sigma} S^{Q}=W_{\mathrm{id}} S^{Q}$. Applying Proposition 3.4 we see there is a maximal ideal $M$ of $S$ such that $M \supseteq Q$ and $M W_{\sigma}$ is a maximal ideal of $W_{\sigma}$. But $M W_{\sigma}$ contains $Q W_{\sigma}$, so $M W_{\sigma}=Q W_{\sigma}$ by the maximality of $Q W_{\sigma}$. Hence $Q=M$ is maximal. We infer that either $J_{\sigma}=W_{\sigma}$ or $J_{\sigma}=\bigcap Q W_{\sigma}$ where the intersection is over those maximal ideals $Q$ of $S$ such that $Q W_{\sigma}$ is a maximal ideal of $W_{\sigma}$ and $f\left(\sigma, \sigma^{-1}\right) \notin Q W_{\text {id }}$.

Now let $I=\sum_{\sigma \in G} J_{\sigma} x_{\sigma}$. We begin by showing that $I$ is an ideal of $B$. To see that it is a right ideal it suffices to show that for all $\sigma, \tau \in G$, $J_{\sigma} x_{\sigma} W_{\tau} x_{\tau} \subseteq J_{\sigma \tau} x_{\sigma \tau}$. This is equivalent to $J_{\sigma} W_{\tau}^{\sigma} f(\sigma, \tau) \subseteq J_{\sigma \tau}$. First observe that $J_{\sigma} W_{\tau}^{\sigma} f(\sigma, \tau) \subseteq W_{\sigma \tau}$. Hence if $J_{\sigma \tau}=W_{\sigma \tau}$ the inclusion is clear. We may thus assume $J_{\sigma \tau}=\bigcap Q W_{\sigma \tau}$ where the intersection is over those maximal ideals $Q$ of $S$ such that $Q W_{\sigma \tau}$ is a maximal ideal of $W_{\sigma \tau}$ and $f\left(\sigma \tau, \tau^{-1} \sigma^{-1}\right) \notin Q W_{\text {id }}$. Hence to show $J_{\sigma} W_{\tau}^{\sigma} f(\sigma, \tau) \subseteq J_{\sigma \tau}$ we need to show that if $Q$ is a maximal ideal of $S$ such that $Q W_{\sigma \tau}$ is maximal in $W_{\sigma \tau}$ and $f\left(\sigma \tau, \tau^{-1} \sigma^{-1}\right) \notin Q W_{\text {id }}$, then $J_{\sigma} W_{\tau}^{\sigma} f(\sigma, \tau) \subseteq Q W_{\sigma \tau}$. This last inclusion is equivalent to $J_{\sigma} W_{\tau}^{\sigma} f(\sigma, \tau) S_{Q} \subseteq Q S_{Q}$.

So assume $Q$ is chosen as above. Let $P$ be the unique height $n-1$ prime of $S$ such that $P \subseteq Q$. If $f\left(\sigma \tau, \tau^{-1} \sigma^{-1}\right) \in P W_{\text {id }}$, then by Lemma 3.2, $W_{\sigma \tau} S^{P}=$ $S_{P}$, contradicting the fact that $W_{\sigma \tau} \subseteq S_{Q}$. Hence we have $f\left(\sigma \tau, \tau^{-1} \sigma^{-1}\right) \notin$ $P W_{\text {id }}$.

Now if $f\left(\sigma, \sigma^{-1}\right) \notin Q W_{\text {id }}$, then in particular $f\left(\sigma, \sigma^{-1}\right) \notin P W_{\text {id }}$ and so by Lemma 3.2 we have $W_{\sigma} S_{Q}=W_{\text {id }} S_{Q}=S_{Q}$. Moreover $J_{\sigma} S_{Q} \subseteq Q S_{Q}$. Hence $J_{\sigma} W_{\tau}^{\sigma} f(\sigma, \tau) S_{Q} \subseteq W_{\tau}^{\sigma} f(\sigma, \tau) Q S_{Q}$. But $W_{\tau}^{\sigma} f(\sigma, \tau) \subseteq W_{\sigma \tau} \subseteq S_{Q}$, so we get the desired inclusion.

Now assume $f\left(\sigma, \sigma^{-1}\right) \notin Q W_{\text {id }}$. Let $P=P_{n-1} \supseteq P_{n-2} \supseteq \cdots \supseteq P_{1} \supseteq$ $P_{0}=0$ be the chain of prime ideals contained in $P$. Assume $j$ is the unique integer such that $f\left(\sigma, \sigma^{-1}\right) \in P_{j} W_{\text {id }}-P_{j-1} W_{\text {id }}$. If $J_{\sigma}=W_{\sigma}$ when $J_{\sigma} S_{Q}=$ $W_{\sigma} S_{Q}=S_{P_{j}}$ by Proposition 3.3. If $J_{\sigma} \neq W_{\sigma}$ then we know $J_{\sigma}=\bigcap N W_{\sigma}$ where the intersection is over those maximal ideals $N$ of $S$ such that $N W_{\sigma}$ is a maximal ideal of $W_{\sigma}$ and $f\left(\sigma, \sigma^{-1}\right) \notin N W_{\text {id }}$. Because $f\left(\sigma, \sigma^{-1}\right) \in P_{j} W_{\text {id }}$ we know that if $N$ is such a maximal ideal then $N$ does not contain $P_{j}$. In particular $J_{\sigma} \supseteq \prod N$ where the product is over those maximal ideals and so $J_{\sigma} S_{Q}=J_{\sigma} W_{\sigma} S_{Q}=J_{\sigma} S_{P_{j}} \supseteq\left(\prod N\right) S_{P_{j}}=S_{P_{j}}$ because otherwise some $N$ would be contained in $P_{j}$. Hence in either case we have $J_{\sigma} S_{Q}=S_{P_{j}}$ and so 


$$
J_{\sigma} W_{\tau}^{\sigma} f(\sigma, \tau) S_{Q}=W_{\tau}^{\sigma} f(\sigma, \tau) S_{P_{j}}=W_{\tau}^{\sigma} f(\sigma, \tau) f\left(\sigma \tau, \tau^{-1} \sigma^{-1}\right) S_{P_{j}},
$$

because $f\left(\sigma \tau, \tau^{-1} \sigma^{-1}\right) \notin P_{j} W_{\text {id }}$. Now we apply the following cocycle identity:

$$
f^{\sigma}\left(\tau, \tau^{-1} \sigma^{-1}\right) f\left(\sigma, \sigma^{-1}\right)=f(\sigma, \tau) f\left(\sigma \tau, \tau^{-1} \sigma^{-1}\right) .
$$

It follows that

$$
W_{\tau}^{\sigma} f(\sigma, \tau) f\left(\sigma \tau, \tau^{-1} \sigma^{-1}\right) S_{P_{j}}=W_{\tau}^{\sigma} f^{\sigma}\left(\tau, \tau^{-1} \sigma^{-1}\right) f\left(\sigma, \sigma^{-1}\right) S_{P_{j}} .
$$

But $f\left(\sigma, \sigma^{-1}\right) \in P_{j} S_{P_{j}}$ and $W_{\tau}^{\sigma} f^{\sigma}\left(\tau, \tau^{-1} \sigma^{-1}\right) \subseteq W_{\sigma^{-1}}^{\sigma}$. Moreover $W_{\sigma^{-1}}^{\sigma} \subseteq S_{P_{j}}$ by part (b) of Lemma 3.2. Thus

$$
W_{\tau}^{\sigma} f^{\sigma}\left(\tau, \tau^{-1} \sigma^{-1}\right) f\left(\sigma, \sigma^{-1}\right) S_{P_{j}} \subseteq P_{j} S_{P_{j}} \subseteq Q S_{Q}
$$

as desired.

The computations to show $I$ is a left ideal are similar and will be omitted.

Having shown $I$ is an ideal we proceed to show $I=J(B)$. Because $B$ is a valuation ring we know $J(B)$ is the unique maximal ideal of $B$, so $I \subseteq J(B)$. Moreover $J(B)$ is an $S$ - $S$ bimodule, so we can write $J(B)=\sum_{\sigma \in G} T_{\sigma} x_{\sigma}$ for some ideals $T_{\sigma}$ in $W_{\sigma}$. We have $T_{\sigma} \supseteq J_{\sigma}$ for all $\sigma \in G$, and we want to show equality.

First observe that

$$
J_{\text {id }}=\left\{k \in W_{\text {id }} \mid k f(\text { id, id }) \in J\left(W_{\text {id }}\right)\right\}=J\left(W_{\text {id }}\right) .
$$

Because $T_{\text {id }}$ consists of quasiregular elements in $W_{\text {id }}$ it follows that $T_{\text {id }} \subseteq$ $J\left(W_{\text {id }}\right)$. Hence $T_{\text {id }}=J_{\text {id }}$.

Now let $\sigma \in G$. If $J_{\sigma}=W_{\sigma}$ then certainly $T_{\sigma}=J_{\sigma}$. Hence we may assume $J_{\sigma} \neq W_{\sigma}$ and so $J_{\sigma}=\bigcap Q W_{\sigma}$ where the intersection is over those maximal ideals $Q$ of $S$ such that $Q W_{\sigma}$ is maximal in $W_{\sigma}$ and $f\left(\sigma, \sigma^{-1}\right) \notin$ $Q W_{\text {id }}$. Let $Q$ be such a maximal ideal. Now because $J(B)$ is an ideal we have $T_{\sigma} x_{\sigma} x_{\sigma^{-1}} \subseteq T_{\text {id }} \subseteq J\left(W_{\text {id }}\right)$ and so $T_{\sigma} f\left(\sigma, \sigma^{-1}\right) \subseteq J\left(W_{\text {id }}\right)$. Moreover if $Q$ is a maximal ideal in $S$ such that $Q W_{\sigma}$ is maximal in $W_{\sigma}$, then $Q W_{\text {id }}$ is a maximal ideal of $W_{\text {id }}$. Hence $J\left(W_{\text {id }}\right) \subseteq J_{\sigma}$ and so $T_{\sigma} f\left(\sigma, \sigma^{-1}\right)^{2} \subseteq J\left(W_{\sigma}\right) \subseteq Q W_{\sigma}$. But $f\left(\sigma, \sigma^{-1}\right)^{2} \notin Q W_{\sigma}$ so $T_{\sigma} \subseteq Q W_{\sigma}$. Because $Q$ was arbitrary, we see that $T_{\sigma} \subseteq J_{\sigma}$, as desired.

3.2. Proof of part (b) of the theorem. If the height of $Q_{1}$ is less than $n$ then the result follows by induction. Hence we may assume that $Q_{1}$ and $Q_{2}$ are maximal ideals of $S$. Let $Q_{1}, Q_{2}, \ldots, Q_{r}, Q_{r+1}, \ldots, Q_{m}$ be all the maximal ideals of $S$ and assume $Q_{1}, Q_{2}, \ldots, Q_{r}$ are the ones that belong to $B$. By Proposition 3.4 we know $W_{\text {id }}=\bigcap_{i=1}^{r} S_{Q_{i}}$. We also know from part (a) of the theorem that

$$
J(B)_{\mathrm{id}}=J\left(W_{\mathrm{id}}\right)=\bigcap_{i=1}^{r} Q_{i} W_{\mathrm{id}} .
$$

Let $T=\prod_{i=2}^{m} Q_{i}$. Then $T W_{\mathrm{id}}=\prod_{i=2}^{r} Q_{i} W_{\mathrm{id}}$ because if $i>r$ then $Q_{i} W_{\mathrm{id}}=$ $W_{\text {id }}$. It follows that $T$ is not contained in $J\left(W_{\text {id }}\right)$. Because $B$ is primary we infer that $B T B=B$. Computing the identity component of $B T B$ we see that we must have $\sum_{\sigma \in G} W_{\sigma} x_{\sigma} T W_{\sigma^{-1}} x_{\sigma^{-1}}=W_{\text {id }}$. 
For each $i, i=1,2, \ldots, m$, there is an element $h_{i} \in G$ such that $Q_{1}^{h_{i}}=$ $Q_{i}$. Let $D=D\left(Q_{1}\right)$. The elements $h_{1}, h_{2}, \ldots, h_{m}$ form a set of left coset representatives of $D$ in $G$. Hence we can write

$$
\begin{aligned}
W_{\text {id }} & =\sum_{\sigma \in G} W_{\sigma} x_{\sigma} T W_{\sigma^{-1}} x_{\sigma^{-1}}=\sum_{\sigma \in G} W_{\sigma} T^{\sigma} W_{\sigma^{-1}}^{\sigma} f\left(\sigma, \sigma^{-1}\right) \\
& =\sum_{i=1}^{m} \sum_{d \in D} W_{h_{i} d} T^{h_{i} d}\left(W_{\left(h_{i} d\right)^{-1}}^{h_{d} d} f\left(h_{i} d,\left(h_{i} d\right)^{-1}\right)\right) .
\end{aligned}
$$

Note that for all $d \in D, T^{h_{i} d}=T^{h_{i}}$ because $T^{d}=T$. Also observe that if $i \neq 2$ then $T^{h_{i}} \subseteq Q_{2}$ and so

$$
\sum_{d \in D} W_{h_{i} d} T^{h_{i} d}\left(W_{\left(h_{i} d\right)^{-1}}^{h_{i} d} f\left(h_{i} d,\left(h_{i} d\right)^{-1}\right)\right) \subseteq Q_{2} W_{\mathrm{id}}
$$

because for every $\sigma \in G, W_{\sigma} W_{\sigma^{-1}}^{\sigma} f\left(\sigma, \sigma^{-1}\right) \subseteq W_{\text {id }}$.

Now assume that for all $d \in D, f\left(h_{2} d,\left(h_{2} d\right)^{-1}\right) \in Q_{1}^{h_{2}} \quad\left(=Q_{2}\right)$. We claim that it follows that

$$
\sum_{d \in D} W_{h_{2} d} T^{h_{2} d}\left(W_{\left(h_{2} d\right)^{-1}}^{h_{2} d} f\left(h_{2} d,\left(h_{2} d\right)^{-1}\right)\right) \subseteq Q_{2} W_{\mathrm{id}} .
$$

If so then $B T B \subseteq Q_{2} W_{\text {id }}$, a contradiction. It will then follow that for some $d \in D, f\left(h_{2} d,\left(h_{2} d\right)^{-1}\right) \notin Q_{1}^{h_{2}}=Q_{1}^{h_{2} d}$ and so that

$$
f\left(\left(h_{2} d\right)^{-1}, h_{2} d\right)=f^{\left(h_{2} d\right)^{-1}}\left(h_{2} d,\left(h_{2} d\right)^{-1}\right) \notin Q_{1} .
$$

Because $Q_{1}^{h_{1} d}=Q_{2}$ that will finish the proof.

To prove the claim it suffices to show that for each $d \in D$

$$
W_{h_{2} d} T^{h_{2} d}\left(W_{\left(h_{2} d\right)^{-1}}^{h_{2} d} f\left(h_{2} d,\left(h_{2} d\right)^{-1}\right)\right) \subseteq Q_{2} W_{\text {id }},
$$

or equivalently that

$$
W_{h_{2} d} T^{h_{2} d}\left(W_{\left(h_{2} d\right)^{-1}}^{h_{2} d} f\left(h_{2} d,\left(h_{2} d\right)^{-1}\right)\right) S_{Q_{2}} \subseteq Q_{2} S_{Q_{2}} .
$$

To simplify the notation let $Q=Q_{2}$. Let $Q=P_{n} \supseteq P_{n-1} \supseteq \cdots \supseteq P_{1} \supseteq P_{0}=0$ be the chain of prime ideals contained in $Q$. Let $d \in D$ and let $j$ be the unique integer such that $f\left(h_{2} d,\left(h_{2} d\right)^{-1}\right) \in P_{j} W_{\text {id }}-P_{j-1} W_{\text {id }}$. Let $\tau=h_{2} d$. We need to show $W_{\tau} T^{\tau} W_{\tau^{-1}}^{\tau} f\left(\tau, \tau^{-1}\right) S_{Q} \subseteq Q S_{Q}$. We have $f\left(\tau, \tau^{-1}\right) \subseteq P_{j} W_{\text {id }}$ and by Proposition 3.3 $W_{\tau} S_{Q}=S_{P_{j}}$. Moreover $W_{\tau^{-1}}^{\tau} \subseteq S_{P_{j}}$ by Lemma 3.2. Hence $W_{\tau} T^{\tau} W_{\tau^{-1}}^{\tau} f\left(\tau, \tau^{-1}\right) S_{Q} \subseteq P_{j} S_{P_{j}}$. But $P_{j} S_{P_{j}} \subseteq Q S_{Q}$, so we are done.

Corollary 3.5. Let $P$ be a prime of height $i<n$ belonging to $B$ and let $T=$ $\{Q \mid Q$ is a prime of height $i+1$ belonging to $B$ and containing $P\}$.

(a) The group $H(P)$ acts transitively on $T$. In particular the order of $T$ is the index $(H(P): D(Q) \cap H(P))$, where $Q$ is any element of $T$.

(b) If $P^{\prime}$ is another height $i$ prime belonging to $B$ and $T^{\prime}=\{Q \mid Q$ is a prime of height $i+1$ belonging to $B$ and containing $\left.P^{\prime}\right\}$ then there is an element $\sigma \in G$ such that $f\left(\sigma^{-1}, \sigma\right) \notin P W_{\mathrm{id}}$ and $T^{\prime}=T^{\sigma}$.

Proof. (a) If $Q \in T$ and $\sigma \in H(P)$ then $f\left(\sigma, \sigma^{-1}\right) \notin P W_{\text {id }}$ so by Lemma 3.2 we know $W_{\text {id }} \subseteq S_{Q^{\sigma^{-1}}}$. Hence the group $H(P)$ acts on $T$. Moreover if 
$Q_{1}$ and $Q_{2}$ are in $T$ then by the theorem there is an element $\sigma$ in $G$ such that $f\left(\sigma^{-1}, \sigma\right) \notin Q_{1} W_{\text {id }}$ and $Q_{2}=Q_{1}^{\sigma}$. Because $P$ is the unique prime of height $i$ contained in $Q_{1}$ and the unique prime of height $i$ contained in $Q_{2}$ it follows that $P^{\sigma}=P$, that is $\sigma \in D(P)$. But $f\left(\sigma, \sigma^{-1}\right)=f^{\sigma}\left(\sigma^{-1}, \sigma\right) \notin$ $Q_{1}^{\sigma} W_{\mathrm{id}}=Q_{2} W_{\text {id }}$, so in particular $f\left(\sigma, \sigma^{-1}\right) \notin P W_{\text {id }}$. Hence $\sigma \in H(P)$. The last statement is immediate.

(b) By Theorem 3.1 there is an element $\sigma \in G$ such that $f\left(\sigma^{-1}, \sigma\right) \notin P W_{\text {id }}$ and $P^{\prime}=P^{\sigma}$. By Lemma 3.2 it follows immediately that every element of $T^{\sigma}$ belongs to $B$, so $T^{\sigma} \subseteq T^{\prime}$. The opposite inclusion follows by considering $f\left(\sigma, \sigma^{-1}\right)=f^{\sigma}\left(\sigma^{-1}, \sigma\right)$.

Corollary 3.6. (a) If $P$ is a prime of height $i<n$ that belongs to $B$, and $Q$ is a prime of height $i+1$ that belongs to $B$ and contains $P$, then there is a set of right coset representatives of $D(Q) \cap H(P)$ in $H(P)$ such that for each representative $g, f\left(g, g^{-1}\right) \notin Q$.

(b) Let $P$ be a prime ideal of height $i<n$ that belongs to $B$. Let $H=H(P)$. The ring $W_{\mathrm{id}} S^{P} \cap K^{H}$ is a valuation ring of rank $i$. Moreover if $\rho$ is the prime ideal in $S^{H}$ of height $i$ such that $W_{\mathrm{id}} S^{P} \cap K^{H}=\left(S^{H}\right)_{\rho}$, then the prime ideals in $S$ of height $i+1$ that belong to $B$ and contain $P$ are precisely those that lie over $\rho$.

Proof. (a) This is an easy consequence of part (a) of Corollary 3.5.

(b) This is also a consequence of part (a) of Corollary 3.5: In the notation of that corollary, because $H$ acts transitively on $T$, it follows that all the prime ideals in $T$ lie over the same prime ideal in $S^{H}$. If we let $\rho$ denote that prime ideal in $S^{H}$, then $T$ consists of precisely the primes of $S$ lying over $\rho$. But $W_{\sigma} S^{P}=\bigcap_{Q \in T} S_{Q}$ and so $W_{\sigma} S^{P} \cap K^{H}=\left(S^{H}\right)_{\rho}$.

The result of Corollary 3.6 and [H, Corollary 3.11] lead one to suspect that there should be a Dubrovin valuation ring "involved" with $B$ whose center is $W_{\text {id }} S^{P} \cap K^{H}$. We are now headed for such a result. Let $P$ be a prime of height $i<n$ belonging to $B$. Let $D=D(P)$ and let $H=H(P)$. Let $B^{\prime}=\sum_{\sigma \in D}\left(W_{\sigma} S^{P}\right) x_{\sigma}$ and let $B^{\prime \prime}=\sum_{\sigma \in H}\left(W_{\sigma} S^{P}\right) x_{\sigma}$. Similarly if $M$ is a maximal ideal of $S$ belonging to $B$ and $E=D(M)$, let $C=\sum_{\sigma \in E}\left(W_{\sigma} S_{M}\right) x_{\sigma}$.

Proposition 3.7. The rings $B^{\prime}, B^{\prime \prime}$, and $C$ are Dubrovin.

Proof. We proceed by induction on $n$, the rank of $B$. If the rank of $B$ is one, then the rank of $P$ must be zero, so $B^{\prime}=B^{\prime \prime}=B$. The fact that $C$ is Dubrovin is the content of [H, Corollary 3.11]. Hence we may assume the rank of $B$ is greater than one.

We begin with $B^{\prime}$. If $P$ is a prime of rank $i<n-1$ then for all $\sigma \in G$ $W_{\sigma} S^{P}=W_{\sigma} V_{n-1} S^{P}$ and so the result follows by induction applied to $B_{n-1}$. Hence we may assume $P$ is a prime of rank $n-1$. It follows that

$$
C^{\prime}=B^{\prime} V_{n-1}=\sum_{\sigma \in D}\left(W_{\sigma} V_{n-1} S^{P}\right) x_{\sigma}=\sum_{\sigma \in D}\left(W_{\sigma} S_{P}\right) x_{\sigma}
$$

is Dubrovin by induction. Moreover we claim $J\left(C^{\prime}\right) \subseteq B^{\prime}$ : We know by Theorem 3.1 that

$$
J\left(C^{\prime}\right)=\sum_{\sigma \in H}\left(P S_{P}\right) x_{\sigma}+\sum_{\substack{\sigma \in D \\ \sigma \notin H}}\left(W_{\sigma} S_{P}\right) x_{\sigma} .
$$


But if $\sigma \notin H$ then $W_{\sigma} S^{P}=W_{\sigma} S_{P}$ by Lemma 3.2 and if $\sigma \in H$ then $P S_{P} \subseteq$ $M S_{M}$ for every maximal ideal of $S$ that contains $P$. Hence if $\sigma \in H$, then $P S_{P} \subseteq S^{P} \subseteq W_{\sigma} S^{P}$. This proves the claim.

Hence by $\left[D_{2}, \S 1\right.$, Proposition 2] it suffices to show $\widetilde{B}^{\prime}=B^{\prime} / J\left(C^{\prime}\right)$ is a valuation ring. To do this we first consider the valuation rings $\widetilde{B}=B / J\left(B_{n-1}\right) \subseteq$ $\bar{B}_{n-1}=B_{n-1} / J\left(B_{n-1}\right)$. Note that by Theorem 3.1, $J\left(B_{n-1}\right)_{\mathrm{id}}=J\left(W_{\mathrm{id}} V_{n-1}\right)$. Hence

$$
\begin{aligned}
\bar{B}_{n-1} & =\overline{W_{\mathrm{id}} V_{n-1}}=W_{\mathrm{id}} V_{n-1} / J\left(W_{\mathrm{id}} V_{n-1}\right) \\
& =\bigoplus \sum_{i=1}^{k}\left(W_{\mathrm{id}} V_{n-1}\right) /\left(P_{i} W_{\mathrm{id}} V_{n-1}\right),
\end{aligned}
$$

where $P=P_{1}, P_{2}, \ldots, P_{k}$ are the primes of height $n-1$ that belong to $B$. Let $e$ be the minimal idempotent in $\overline{W_{\text {id }} V_{n-1}}$ corresponding to

$$
\left(W_{\mathrm{id}} V_{n-1}\right) /\left(P W_{\mathrm{id}} V_{n-1}\right) \text {. }
$$

Next observe that

$$
J\left(W_{\mathrm{id}} V_{n-1}\right)=\bigcap_{i=1}^{k} P_{i} W_{\mathrm{id}} V_{n-1} \subseteq \bigcap_{i=1}^{k} P_{i} S_{P_{i}} \subseteq \bigcap M S_{M}
$$

where this last intersection is over all the maximal ideals that belong to $B$. Hence $J\left(W_{\text {id }} V_{n-1}\right) \subseteq J\left(W_{\text {id }}\right)$. Moreover

$$
W_{\text {id }} / J\left(W_{\mathrm{id}} V_{n-1}\right)=\bigoplus \sum_{i=1}^{k} W_{\mathrm{id}} / P_{i} W_{\mathrm{id}}
$$

and for each $i, W_{\mathrm{id}} / P_{i} W_{\mathrm{id}}$ is a Dedekind domain with field of fractions

$$
\left(W_{\mathrm{id}} V_{n-1}\right) /\left(P_{i} W_{\mathrm{id}} V_{n-1}\right) \text {. }
$$

In particular note that $e \in W_{\text {id }} / P_{i} W_{\text {id }} \subseteq \widetilde{B}$. Because $\widetilde{B}$ is a valuation ring in the simple algebra $\bar{B}_{n-1}$, it follows from [ $D_{1}, \S 1$, Theorem 7] that $e \widetilde{B} e$ is a valuation ring in the simple algebra $e \bar{B}_{n-1} e$.

We want to compute $e \widetilde{B} e$ and $e \bar{B}_{n-1} e$. To simplify notation let $T_{\sigma}=$ $W_{\sigma} V_{n-1}$ and let $I_{\sigma}=J\left(B_{n-1}\right)_{\sigma}$, the $\sigma$-component of the radical of $B_{n-1}$. We have

$$
e \bar{B}_{n-1} e=\sum_{\sigma \in G} e\left(T_{\sigma} / I_{\sigma}\right) \sigma(e) x_{\sigma} .
$$

If $\sigma \notin D$ then $\sigma(e) \neq e$ and so $\sigma(e) e=0$. Hence

$$
e \bar{B}_{n-1} e=\sum_{\sigma \in D} e\left(T_{\sigma} / I_{\sigma}\right) \sigma(e) x_{\sigma} .
$$

Moreover we claim if $\sigma \notin H$ then $e\left(T_{\sigma} / I_{\sigma}\right)=0$ : If $I_{\sigma}=T_{\sigma}$ then the claim is certainly true. If $I_{\sigma} \neq T_{\sigma}$ then by the description of $I_{\sigma}$ given in the proof of part (a) of Theorem 3.1 we know that $I_{\sigma}=\bigcap Q T_{\sigma}$ where the intersection is over those prime ideals $Q$ of height $n-1$ that belong to $B$ and for which $f\left(\sigma, \sigma^{-1}\right) \notin Q T_{\sigma}$. But $f\left(\sigma, \sigma^{-1}\right) \in P T_{\sigma}$ and so $P$ is not among those primes. The claim follows. Hence $e \bar{B}_{n-1} e=\sum_{\sigma \in H} e\left(T_{\sigma} / I_{\sigma}\right) x_{\sigma}$. It follows that $e \widetilde{B} e=$ $\sum_{\sigma \in H} e\left(W_{\sigma} / I_{\sigma}\right) x_{\sigma}$. 
We want to show that $\widetilde{B}^{\prime} \cong e \widetilde{B} e$. If so then $\widetilde{B}^{\prime}$ is a valuation ring, as desired. We have $\widetilde{B}^{\prime}=\sum_{\sigma \in H}\left(W_{\sigma} S^{P} / P S_{P}\right) x_{\sigma}$. If $\sigma \in H$ then we know by Proposition 3.3 that $W_{\sigma} \subseteq S_{P}$ and so $P W_{\sigma}$ is a proper ideal of $W_{\sigma}$. Moreover $I_{\sigma} \subseteq P W_{\sigma}$. Hence there is a canonical homomorphism $\phi$ from $W_{\sigma} / I_{\sigma}$ to $S_{P} / P S_{P}$. It is easy to see that $e\left(W_{\sigma} / I_{\sigma}\right)$ is precisely the image of $\phi$. On the other hand, $W_{\sigma} \subseteq W_{\sigma} S^{P} \subseteq S_{P}$ and $P S_{P} \subseteq W_{\sigma} S^{P}$. We have then the following commutative diagram of ring homomorphisms, where $\rho$ is the canonical ring homomorphism from $W_{\sigma} S^{P} / P S_{P}$ to $S_{P} / P S_{P}$.

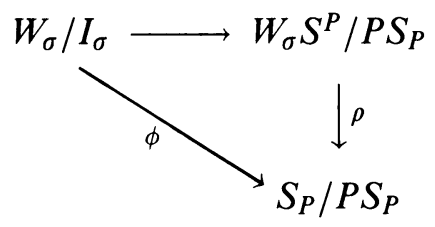

To show that $\widetilde{B}^{\prime}$ is isomorphic to $e \widetilde{B} e$, it suffices to show that in this diagram $\phi$ and $\rho$ have the same image. Hence it is enough to show that the map from $W_{\sigma} / I_{\sigma}$ to $W_{\sigma} S^{P} / P S_{P}$ is surjective. Thus we are reduced to showing that $W_{\sigma}+$ $P S_{P}=W_{\sigma} S^{P}$. We have $W_{\sigma} \subseteq W_{\sigma}+P S_{P} \subseteq W_{\sigma} S^{P}$. Because $W_{\sigma} \subseteq S_{P}$ we see that $P S_{P}$ is a $W_{\sigma}$-submodule of $S_{P}$ and so $W_{\sigma}+P S_{P}$ is a ring. By the properties of Prüfer rings, we know that $W_{\sigma}+P S_{P}=\bigcap S_{Q}$ where the intersection is over those prime ideals $Q$ of $S$ such that $W_{\sigma}+P S_{P} \subseteq S_{Q}$. Moreover $W_{\sigma} S^{P}=\cap S_{M}$ where the intersection is over those maximal ideals $M$ of $S$ such that $M \supseteq P$ and $W_{\sigma} \subseteq S_{M}$. Hence it suffices to show that if $Q$ is a prime ideal of $S$ such that $P S_{P} \subseteq S_{Q}$, then $Q \supseteq P$. To see this statement we may assume $Q \neq P$. If $P S_{P} \subseteq S_{Q}$, then $Q P S_{P} \subseteq Q S_{Q}$. But $Q S_{P}=S_{P}$ because $Q$ is not contained in $P$. Hence $P S_{P} \subseteq Q S_{Q}$, so $P=P S_{P} \cap S \subseteq$ $Q S_{Q} \cap S=Q$. Hence we have shown that $\widetilde{B}^{\prime}$ is isomorphic to $e \widetilde{B} e$ and so $\widetilde{B}^{\prime}$ is a valuation ring. As we have seen it follows that $B^{\prime}$ is a valuation ring.

It is now easy to see that $B^{\prime \prime}$ is also a valuation ring: We have

$$
B^{\prime \prime} \subseteq C^{\prime \prime}=\sum_{\sigma \in H}\left(W_{\sigma} S^{P} V_{n-1}\right) x_{\sigma}=\sum_{\sigma \in H}\left(W_{\sigma} S_{P}\right) x_{\sigma}=\sum_{\sigma \in H} S_{P} x_{\sigma} .
$$

The algebra $C^{\prime \prime}$ is Azumaya, hence a valuation ring. Moreover $J\left(C^{\prime \prime}\right)=$ $\sum_{\sigma \in H} P S_{P} x_{\sigma}$. To show $B^{\prime \prime}$ is a valuation ring it therefore suffices to show that the quotient ring $B^{\prime \prime} / J\left(C^{\prime \prime}\right)$ is a valuation ring. But clearly $B^{\prime \prime} / J\left(C^{\prime \prime}\right)=\widetilde{B}^{\prime}$, which we just proved is a valuation ring.

Finally we need to show $C=\sum_{\sigma \in E}\left(W_{\sigma} S_{M}\right) x_{\sigma}$ is a valuation ring. Let $P$ be the unique prime of height $n-1$ contained in $M$. Again we consider

$$
C^{\prime}=C V_{n-1}=\sum_{\sigma \in E}\left(W_{\sigma} S_{M} V_{n-1}\right) x_{\sigma}=\sum_{\sigma \in E}\left(W_{\sigma} S_{P}\right) x_{\sigma} \subseteq \sum_{\sigma \in D}\left(W_{\sigma} S_{P}\right) x_{\sigma} .
$$

By the induction hypothesis, $\sum_{\sigma \in D}\left(W_{\sigma} S_{P}\right) x_{\sigma}$ is a valuation ring and so by Proposition 2.19 the ring $C^{\prime}=\sum_{\sigma \in E} W_{\sigma} S_{P} x_{\sigma}$ is a valuation ring. Hence we know

$$
J\left(C^{\prime}\right)=\sum_{\substack{\sigma \in E \\ \sigma \in H}} P S_{P} x_{\sigma}+\sum_{\substack{\sigma \in E \\ \sigma \notin H}} W_{\sigma} S_{P} x_{\sigma} .
$$


The quotient ring $C / J\left(C^{\prime}\right)$ is

$$
\sum_{\substack{\sigma \in E \\ \sigma \in H}}\left(W_{\sigma} S_{M} / P S_{P}\right) x_{\sigma}=\sum_{\substack{\sigma \in E \\ \sigma \in H}}\left(S_{M} / P S_{P}\right) x_{\sigma}
$$

It suffices to show $C / J\left(C^{\prime}\right)$ is a valuation ring.

Recall that

$$
\widetilde{B}^{\prime}=\sum_{\sigma \in H}\left(W_{\sigma} S^{P} / P S_{P}\right) x_{\sigma}=\sum_{\sigma \in H}\left(W_{\mathrm{id}} S^{P} / P S_{P}\right) x_{\sigma},
$$

by Lemma 3.2. Let $M=M_{1}, M_{2}, \ldots, M_{m}$ be the maximal ideals of $S$ that contain $P$ and belong to $B$. Then $W_{\mathrm{id}} S^{P}=\bigcap_{i} S_{M_{i}}$, and so $W_{\mathrm{id}} S^{P} / P S_{P}=$ $\bigoplus \sum_{i} S_{M_{i}} / P S_{M_{i}}$. If we let $f$ be the minimal idempotent in $W_{\mathrm{id}} S^{P} / P S_{P}$ corresponding to $S_{M} / P S_{M}$, then an argument similar to that given above shows that $f \widetilde{B}^{\prime} f$ is isomorphic to $C / J\left(C^{\prime}\right)$. It follows that $C / J\left(C^{\prime}\right)$ and hence $C$ are valuation rings.

Corollary 3.8. For every $i \leq n$ if $P$ is a prime of height $i$ that belongs to $B$, then $H(P)$ is a normal subgroup of $D(P) \cap H(Q)$ where $Q$ is the unique prime of height $i-1$ contained in $P$. Moreover, the quotient group $D(P) \cap H(Q) / H(P)$ is cyclic and there is an element $\sigma \in D(P) \cap H(Q)$ such that the following conditions hold:

(i) The coset $\sigma H(P)$ generates $D(P) \cap H(Q) / H(P)$ and $f\left(\sigma, \sigma^{-1}\right) \in P W_{\mathrm{id}}$ $-\left(P W_{\mathrm{id}}\right)^{2}$.

(ii) The partial ordering on $D(P) \cap H(Q) / H(P)$ is the chain $H(P) \leq \sigma H(P)$ $\leq \sigma^{2} H(P) \leq \cdots \leq \sigma^{m-1} H(P)$, where $m=|D(P) \cap H(Q) / H(P)|$.

Proof. Let $D=D(P)$ and $H=H(P)$. The ideal $P W_{\text {id }} V_{i}$ is a maximal ideal of $W_{\text {id }} V_{i}$. By Proposition 3.7, $C=\sum_{\sigma \in D} S_{P} x_{\sigma}$ is a valuation ring. The result now follows from Theorem 2.14.

We end this section with an example designed to display some of the various phenomena we have discussed. Let $k$ be a field of characteristic not two and let $s, t, x, y$ be indeterminates over $k$. Set $F=k(s, t)(x, y)$ and $K=$ $F(\sqrt{1+x}, \sqrt{1+y}, \sqrt{1+2 x})$. Let $\Sigma_{f}$ be the $F$-algebra given by

$$
\Sigma_{f}=\left(\frac{1+x, s}{F}\right) \otimes_{F}\left(\frac{1+y, t}{F}\right) \otimes_{F}\left(\frac{1+2 x, y}{F}\right),
$$

the tensor product of three quaternion algebras. Then $\Sigma_{f}=(K / F, G, f)$ where $G=\langle\sigma, \tau, \rho\rangle$ with

$$
\begin{array}{llll}
\sigma(\sqrt{1+x})=-\sqrt{1+x}, & \sigma(\sqrt{1+y})=\sqrt{1+y}, & \sigma(\sqrt{1+2 x})=\sqrt{1+2 x}, \\
\tau(\sqrt{1+x})=\sqrt{1+x}, & \tau(\sqrt{1+y})=-\sqrt{1+y}, & \tau(\sqrt{1+2 x})=\sqrt{1+2 x} \\
\rho(\sqrt{1+x})=\sqrt{1+x}, & \rho(\sqrt{1+y})=\sqrt{1}, & \rho(\sqrt{1+2 x})=-\sqrt{1+2 x}+y
\end{array}
$$

and the cocycle is given by Table 1 .

Let $V_{1}$ be the $y$-adic valuation ring of $F$ and $V_{2} \subseteq V_{1}$ the $(x, y)$-adic valuation ring of $F$. Let $W_{i}$ be the integral closure of $V_{i}$ in $K$, for $i=$ 1, 2. An easy calculation shows $W_{i}=V_{i}[\sqrt{1+x}, \sqrt{1+y}, \sqrt{1+2 x}]$. Write 
TABLE 1

\begin{tabular}{|c|cccccccc|}
\hline$f$ & 1 & $\sigma$ & $\tau$ & $\rho$ & $\sigma \tau$ & $\sigma \rho$ & $\tau \rho$ & $\sigma \tau \rho$ \\
\hline 1 & 1 & 1 & 1 & 1 & 1 & 1 & 1 & 1 \\
$\sigma$ & 1 & $s$ & 1 & 1 & $s$ & $s$ & 1 & $s$ \\
$\tau$ & 1 & 1 & $t$ & 1 & $t$ & 1 & $t$ & $t$ \\
$\rho$ & 1 & 1 & 1 & $y$ & 1 & $y$ & $y$ & $y$ \\
$\sigma \tau$ & 1 & $s$ & $t$ & 1 & $s t$ & $s$ & $t$ & $s t$ \\
$\sigma \rho$ & 1 & $s$ & 1 & $y$ & $s$ & $s y$ & $y$ & $s y$ \\
$\tau \rho$ & 1 & 1 & $t$ & $y$ & $t$ & $y$ & $t y$ & $t y$ \\
$\sigma \tau \rho$ & 1 & $s$ & $t$ & $y$ & $s t$ & $s y$ & $t y$ & $s t y$ \\
\hline
\end{tabular}

$\Sigma_{f}=\sum_{\gamma \in G} K x_{\gamma}$ be the crossed product algebra determined by $f$. We want to construct for $i=1,2$ valuation rings $B_{i}$ in $\Sigma_{f}$ with $B_{i} \cap F=V_{i}$. Let

$$
\Sigma_{1}=\left(\frac{1+x, s}{F}\right) ; \quad \Sigma_{2}=\left(\frac{1+y, t}{F}\right) ; \quad \Sigma_{3}=\left(\frac{1+2 x, y}{F}\right) .
$$

For each $j$ let $K_{j}=\Sigma_{j} \cap K$ and let $S=W_{2}$.

Because $1+x, s, 1+y, t \in V_{1}^{\times}$,

$$
\left(\frac{1+x, s}{V_{1}}\right) \otimes_{V_{1}}\left(\frac{1+y, t}{V_{1}}\right) \subseteq \Sigma_{1} \otimes_{F} \Sigma_{2}
$$

is Azumaya over $V_{1}$. Now $\bar{V}_{1}=k(s, t)(x)$. Because $1+2 x \in \bar{V}_{1}-\left(\bar{V}_{1}\right)^{2}$ and $v(y) \notin 2 \Gamma_{v_{1}}$, where $v$ is the $y$-adic valuation of $F$, it follows by [JW, Example 4.3] that there is an invariant valuation ring $A$ in $\Sigma_{3}$ extending $V_{1}$. It can be seen that $A=\left(W_{1} \cap K_{3}\right)+\left(W_{1} \cap K_{3}\right) x_{\rho}$. Therefore by [W, Proposition 3.3] we may take $B_{1}$ to be

$$
B_{1}=\left(\frac{1+x, s}{V_{1}}\right) \otimes_{V_{1}}\left(\frac{1+y, t}{V_{1}}\right) \otimes_{V_{1}} A .
$$

It follows that $B_{1}=\sum_{\gamma \in G} W_{1} x_{\gamma}$. Also by [W, Proposition 3.3]

$$
J\left(B_{1}\right)=\left(\frac{1+x, s}{V_{1}}\right) \otimes_{V_{1}}\left(\frac{1+y, t}{V_{1}}\right) \otimes_{V_{1}} J(A),
$$

where

$$
J(A)=\left(J\left(W_{1}\right) \cap K_{3}\right)+\left(W_{1} \cap K_{3}\right) x_{\rho} .
$$

Hence

$$
J\left(B_{1}\right)=\sum_{\gamma \in\langle\sigma, \tau\rangle} J\left(W_{1}\right) x_{\gamma}+\sum_{\gamma \notin\langle\sigma, \tau\rangle} W_{1} x_{\gamma} .
$$

For $B_{2}$ again we see $\left((1+x, s) / V_{2}\right) \otimes_{V_{2}}\left((1+y, t) / V_{2}\right)$ is Azumaya. For $\Sigma_{3}$ we see that because $A / J(A)=\bar{V}_{1}(\sqrt{1+2 x})$ and $V_{2} / J\left(V_{1}\right)$ extends in two ways to $A / J(A)$, it follows that if $C$ is the preimage in $A$ of one of these two 
extensions, then $C$ is a (noninvariant) total valuation ring in $\Sigma_{3}$. Hence by [W, Proposition 3.3]

$$
B_{2}=\left(\frac{1+x, s}{V_{2}}\right) \otimes_{V_{2}}\left(\frac{1+y, t}{V_{2}}\right) \otimes_{V_{2}} C
$$

is a valuation ring of $\Sigma_{f}$ with $B_{2} \cap F=V_{2}$ and $B_{2} \subseteq B_{1}$. One can check that $C=\left(S_{M} \cap K_{3}\right)+\left(S_{P} \cap K_{3}\right) x_{\rho}$ for some maximal ideal $M \supseteq P$ of $S$.

Because $V_{1}$ extends uniquely to $F(\sqrt{1+x}, \sqrt{1+2 x})$ but not to $F(\sqrt{1+y})$ we have $D(P)=\langle\sigma, \rho\rangle$ and $H(P)=\langle\sigma\rangle$. Because $V_{2}$ is completely split in $K, D(M)=\langle 1\rangle$ and so $H(M)=\langle 1\rangle$. From the description of $B_{1}$ we see that

$$
B_{2}=\sum_{\gamma \in\langle\sigma, \tau\rangle} W_{\mathrm{id}} x_{\gamma}+\sum_{\gamma \notin\langle\sigma, \tau\rangle} W_{\rho} x_{\gamma} .
$$

To determine $W_{\text {id }}$ we see that because $D(M)=\langle 1\rangle$ and $G\left(K / K_{3}\right)=\langle\sigma, \tau\rangle$, $S_{M} \cap K_{3} \subseteq S_{M} \cap S_{M^{\sigma}} \cap S_{M^{\tau}} \cap S_{M^{\sigma \tau}}$ and so $W_{\mathrm{id}}=S_{M} \cap S_{M^{\sigma}} \cap S_{M^{\tau}} \cap S_{M^{\sigma \tau}}$. Moreover because $J\left(B_{1}\right) \subseteq B_{2}$, the description of $J\left(B_{1}\right)$ forces $W_{1} \subseteq W_{\rho}$ and so $W_{\rho}=W_{1}$. Thus

$$
B_{2}=\sum_{\gamma \in\langle\sigma, \tau\rangle}\left(S_{M} \cap S_{M^{\sigma}} \cap S_{M^{\tau}} \cap S_{M^{\sigma \tau}}\right) x_{\gamma}+\sum_{\gamma \notin\langle\sigma, \tau\rangle} W_{1} x_{\gamma} .
$$

If $J\left(B_{2}\right)=\sum_{\gamma \in G} J_{\gamma} x_{\gamma}$, we see that $J_{\gamma}=W_{1}$ for $\gamma \notin\langle\sigma, \tau\rangle$ because $J\left(B_{1}\right) \subseteq$ $J\left(B_{2}\right)$. If $\gamma \in\langle\sigma, \tau\rangle$ then because $J_{\gamma}=\left\{a \in K \mid a f\left(\gamma, \gamma^{-1}\right) \in J\left(W_{\text {id }}\right)\right\}$ and $f\left(\gamma, \gamma^{-1}\right) \in V_{2}^{\times}$, we have

$$
J_{\gamma}=J\left(W_{\mathrm{id}}\right)=M S_{M} \cap M^{\sigma} S_{M^{\sigma}} \cap M^{\tau} S_{M^{\tau}} \cap M^{\sigma \tau} S_{M^{\sigma \tau}}
$$

and so

$$
J\left(B_{2}\right)=\sum_{\gamma \in\langle\sigma, \tau\rangle} J\left(W_{\mathrm{id}}\right) x_{\gamma}+\sum_{\gamma \notin\langle\sigma, \tau\rangle} W_{1} x_{\gamma} .
$$

If $B_{1}^{\prime \prime}=\sum_{\gamma \in H(P)} W_{1} x_{\gamma}$ and $B_{2}^{\prime \prime}=\sum_{\gamma \in H(P)} S^{P} x_{\gamma}$, where as usual $S^{P}=\bigcap_{M \supseteq P} S_{M}$, then $B_{i}^{\prime \prime}$ is a valuation ring over $V_{i}$ and $\overline{B_{1}}$ is Brauer equivalent to $\overline{B_{1}^{\prime \prime}}=$ $\overline{S_{P}^{H(P)}}=\overline{V_{1}}(\sqrt{1+2 x})$ while $\overline{B_{2}}$ is Brauer equivalent to $\overline{B_{2}^{\prime \prime}}=\overline{S_{M}^{H(M)}}=\overline{V_{2}}$.

\section{REFERENCES}

[AS] S. Amitsur and L. Small, Prime ideals in PI rings, J. Algebra 62 (1980), 358-383.

[BG] H. Brungs and J. Gräter, Extensions of valuation rings in central simple algebras, Trans. Amer. Math. Soc. 317 (1990), 287-302.

[D $\mathrm{D}_{1}$ N. Dubrovin, Noncommutative valuation rings, Trudy Moscov. Mat. Obshch. 45 (1982), 265-280; English transl., Trans. Moscow Math. Soc. 45 (1984), 273-287.

$\left[\mathrm{D}_{2}\right]$ - Noncommutative valuation rings in simple finite-dimensional algebras over a field, Mat. Sb. 123 (1984), 496-509; English transl., Math. USSR-Sb. 51 (1985), 493-505.

[E] O. Endler, Valuation theory, Springer-Verlag, Berlin and New York, 1972.

[H] D. Haile, Crossed-product orders over discrete valuation rings, J. Algebra 105 (1987), 116148.

[JW] B. Jacob and A. Wadsworth, Division algebras over Henselian fields, J. Algebra 128 (1990), 126-179.

[M] P. Morandi, Value functions on central simple algebras, Trans. Amer. Math. Soc. 315 (1989), 605-622. 
[MW] P. Morandi and A. Wadsworth, Integral Dubrovin valuation rings, Trans. Amer. Math. Soc. 315 (1989), 623-640.

[R] R. Ribenboim, Theorie des valuations, Presses Univ. Montréal, Montréal, 1968.

[W] A. Wadsworth, Dubrovin valuation rings and Henselization, Math. Ann. 283 (1989), 301328.

[We] M. Westmoreland, Doctoral dissertation, University of Texas at Austin, 1990.

Department of Mathematics, Indiana University, Bloomington, Indiana 47405

E-mail address: haile@ucs.indiana.edu

Department of Mathematics, New Mexico State University, las Cruces, New Mexico 88003

E-mail address: pmorandi@nmsu.edu 